\title{
The Cryptosporidium parvum Kinome
}

\author{
Jennifer D Artz ${ }^{1 *}$, Amy K Wernimont ${ }^{1}$, Abdellah Allali-Hassani ${ }^{1}$, Yong Zhao ${ }^{1}$, Mehrnaz Amani ${ }^{1}$, Yu-Hui Lin ${ }^{1}$, \\ Guillermo Senisterra', Gregory A Wasney ${ }^{1}$, Oleg Fedorov², Oliver King², Annette Roos², Vlad V Lunin', Wei Qiu', \\ Patrick Finerty $\mathrm{Jr}^{1}$, Ashley Hutchinson ${ }^{1}$, Irene Chau ${ }^{1}$, Frank von Delft², Farrell MacKenzie ${ }^{1}$, Jocelyne Lew ${ }^{1}$, \\ Ivona Kozieradzki ${ }^{1}$, Masoud Vedadi ${ }^{1}$, Matthieu Schapira ${ }^{1}$, Chao Zhang $^{3}$, Kevan Shokat ${ }^{3}$, Tom Heightman ${ }^{2}$ and \\ Raymond $\mathrm{Hui}^{1}$
}

\begin{abstract}
Background: Hundreds of millions of people are infected with cryptosporidiosis annually, with immunocompromised individuals suffering debilitating symptoms and children in socioeconomically challenged regions at risk of repeated infections. There is currently no effective drug available. In order to facilitate the pursuit of anti-cryptosporidiosis targets and compounds, our study spans the classification of the Cryptosporidium parvum kinome and the structural and biochemical characterization of representatives from the CDPK family and a MAP kinase.

Results: The C. parvum kinome comprises over 70 members, some of which may be promising drug targets. These C. parvum protein kinases include members in the AGC, Atypical, CaMK, CK1, CMGC, and TKL groups; however, almost 35\% could only be classified as OPK (other protein kinases). In addition, about 25\% of the kinases identified did not have any known orthologues outside of Cryptosporidium spp. Comparison of specific kinases with their Plasmodium falciparum and Toxoplasma gondii orthologues revealed some distinct characteristics within the C. parvum kinome, including potential targets and opportunities for drug design. Structural and biochemical analysis of 4 representatives of the CaMK group and a MAP kinase confirms features that may be exploited in inhibitor design. Indeed, screening CPCDPK1 against a library of kinase inhibitors yielded a set of the pyrazolopyrimidine derivatives (PP1-derivatives) with $I C_{50}$ values of $<10 \mathrm{nM}$. The binding of a PP1-derivative is further described by an inhibitor-bound crystal structure of CPCDPK1. In addition, structural analysis of CpCDPK4 identified an unprecedented Zn-finger within the CDPK kinase domain that may have implications for its regulation.
\end{abstract}

Conclusions: Identification and comparison of the C. parvum protein kinases against other parasitic kinases shows how orthologue- and family-based research can be used to facilitate characterization of promising drug targets and the search for new drugs.

\section{Background}

More than 58 million children are afflicted annually with diarrheal disease associated with the most prevalent infections of the small intestine, including Escherichia coli, Rotavirus, Giardia lamblia, and Cryptosporidium parvum, which ultimately results in the death of 2.5 million children [1]. C. parvum is an obligate parasite in the same phylum of Apicomplexa as Plasmodium and the same order of Eucoccidiorida as Toxoplasma and Eimeria. It is one of the pathogenic agents responsible

\footnotetext{
* Correspondence: jdartz@gmail.com

'Structural Genomics Consortium, University of Toronto, MaRS South Tower, Floor 7, 101 College St, Toronto, Ontario M5G 1L7, Canada

Full list of author information is available at the end of the article
}

for cryptosporidiosis, a zoonotic and enteric disease. Children in resource-poor settings are particularly at risk, not only with an increased incidence of Cryptosporidium spp. infection, but also with increased acute and long-lasting morbidity. Psychomotor developmental stunting may occur following infection, especially in children under one year of age, with its effects still measurable many years after infection [2,3]. Malnutrition is both a contributing factor and a result of Cryptosporidium spp. infection $[4,5]$. In this environment, malnutrition, immune immaturity, and HIV-infection often synergistically affect the severity of Cryptosporidium spp. infection. This situation, added to socioeconomic isolation of most afflicted regions, has led to marginalization
C Biomed Central 
of cryptosporidiosis as a neglected disease, one that lacks an effective drug [6]. Paromomycin and nitazoxanide are considered only partially effective in otherwise healthy patients, while nitazoxanide is ineffective in AIDS patients [7].

The research efforts to find therapeutics for cryptosporidiosis are scant, relative to resources dedicated to other protozoan diseases, such as malaria. To date, only 61 structures from Cryptosporidium spp. (compared to almost 400 from Plasmodium spp.) have been deposited to the RSCB Protein Databank http://www.rscb.org. In fact, prior to our first work on C. parvum beginning in late 2004, only 2 Cryptosporidium structures had been deposited and released (both dihydrofolate reductasethymidylate synthase). Cryptosporidium structure determination, is arguably a contributing step to the development of effective inhibitors and ultimately drugs. Structural genomics efforts have greatly enhanced the diversity and overall number of presently available structures by contributing over $70 \%$ of all currently available Cryptosporidium structures covering 34 different proteins/domains, while the remaining $30 \%$ of structures (17) only covers 5 different targets. This focus of research on a few targets, leaving many targets underexplored, plagues drug development today $[8,9]$. In addition, to the best of our knowledge, there have been only 4 studies to date in which a Cryptosporidium target and effective inhibitors have been identified and characterized. These include inosine 5'-monophosphate dehydrogenase [10], $S$-adenosylhomocysteine hydrolase [11], nonspecific polyprenyl pyrophosphate synthase (related to farnesyl pyrophosphate synthase) [12] and calciumdependent protein kinase 1 (CDPK1) [13], where the latter two targets were contributed by structural genomics groups. In order to stimulate interest in new Cryptosporidium targets, we have selected for study the C. parvum kinome. As one of the largest protein families in eukaryotic genomes [14] and with many inhibitor libraries commercially available, protein kinases are considered attractive drug targets for human and infectious diseases alike [15]. Already, Plasmodium kinases are the subject of a growing body of research [16-18], as are the Toxoplasma gondii kinases [19]. In contrast, Cryptosporidium parvum PKs ( $C p \mathrm{PK})$ are only incidentally mentioned in publications focusing on Plasmodium or other parasites. In an endeavour to address the void, our study spans the classification of the C. parvum kinome and the structural and biochemical characterization of representatives from the CDPK family and a MAP kinase. Comparison of the $C p P K s$ (protein kinases) with other known parasitic kinases illustrates some of their unique features and demonstrates that there are potential drug targets, as well as opportunities for drug design.

\section{Results and Discussion}

\section{Breakdown of the Cryptosporidium parvum kinome}

Assignment of the protein kinases to their subfamilies was accomplished via clustering of the kinase domain by sequence similarity. Additional information from domains outside of the catalytic domain and from evolutionary conservation was also used to aid in the analysis, culminating in a classification that rests on a hybrid of results. As such, we found 73 protein kinases with intact catalytic triads [20], including those falling into the following categories: AGC, CaMK, CK1, CMGC, TKL, Atypical, and OPK (other protein kinases). Like $P$. falciparum, there are no STE or tyrosine kinases [17], whereas only 1 STE kinase was noted in the $T$. gondii kinome analysis [19]. Of all the protein kinases found, almost a quarter (17) have no predicted orthologues outside of Cryptosporidium spp. The breakdown of the C. parvum kinome is shown in Figure 1 (and also Additional File 1, Table S1 for a summary table and Additional File 2, Table S2 for the alignments).

\section{AGC group}

From the AGC group, 5 protein kinases were clearly identified, including the $3 \mathrm{cAMP}$-dependent protein kinases (PKA) or PKA-like kinases. The CpPKA kinase cgd3_3040 is an orthologue to the P. falciparum and T. gondii PKA kinases, PFI1685w and TGME49_026030, respectively. The CpPKA-like kinases include cgd1_1220 (based on its TgPKA orthologue TGME49_072200) and cgd2_1830 (without predicted orthologues, but anticipated to be PKA-like from its homology with cgd1_1220). Except for CpPKA and its orthologues, which share $\sim 60 \%$ full length sequence identity, these protozoan PKA-like orthologues are quite divergent sharing less than $30 \%$ identity between them. Notably, CpPKA-like kinase (cgd2_1830) is significantly shorter at the $N$-terminus and no GxGxxG motif can be identified (from subdomain I, this glycine triad structurally forms a hairpin around the bound ATP). Two of these apicomplexan PKA-like kinases (cgd1_1220 and TGME49_072200) have large $C$-terminal extensions (> 200 residues) of unknown function. Similar to C. par$v u m, T$. gondii has a proportionally large number of PKA(-like) kinases which includes 6 of the 10 members of the AGC group [19], while 1 PKA kinase of the 5 members of the $P$. falciparum AGC group was identified [17]. Cgd7_120 is the only cAMP-dependent protein kinase regulatory subunit annotated in the Cryptosporidium database, CryptoDB [21], while there are 3 in $T$. gondii (TGME49_042070, TGME49_111300, and TGME49_019070) and 1 in P. falciparum (PFL1110c).

C. parvum contains a single cGMP-dependent protein kinase $(C p \mathrm{PKG}$ ), namely cgd8_750 (an orthologue to 


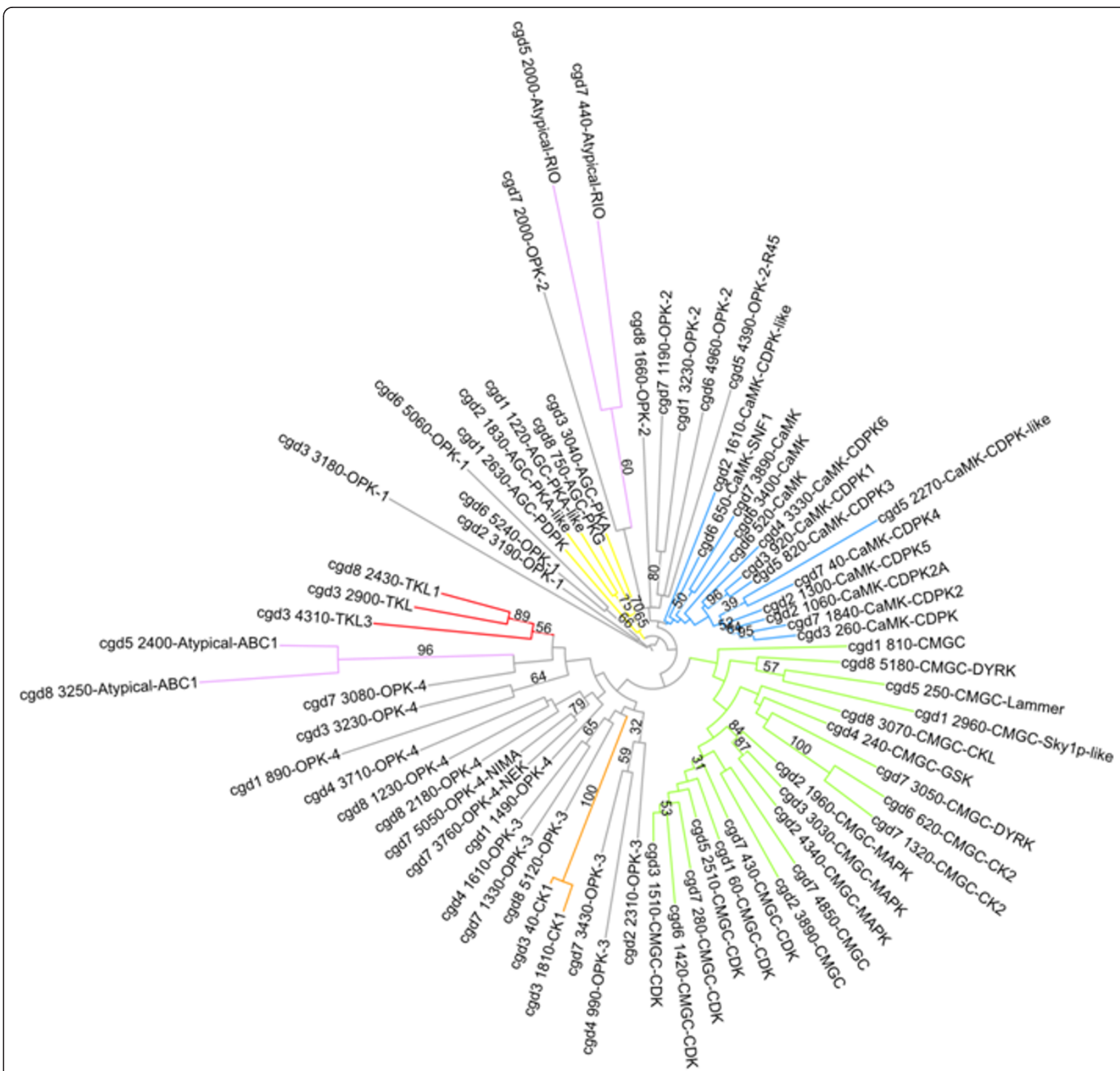

Figure 1 Maximum likelihood tree from a rapid bootstrap analysis describing the classification of the 73 C. parvum protein kinases. The families are colour-coded as follows: AGC (yellow), CK1 (orange), Atypical (pink), CaMK (blue), CMGC (green), TKL (red), and OPK (grey).

PfPKG and TgPKG, PF14_0346 and TGME49_111360, respectively). PfPKG is essential in the blood stage and in gametogenesis of $P$. falciparum infection [22,23]. $C p$ PKG is predicted to have 3 cyclic nucleotide-binding domains (cNMP-BD) upstream of the kinase domain, while PfPKG and TgPKG each have 4 predicted cNMPBDs.

The orthologue to the T. gondii and P. falciparum phosphoinositide-dependent protein kinase (PDPK) (TGME49_068210 and PF11_0227, respectively) is cgd1_2630. These are $230 \%$ identical in sequence, but $C p$ PDPK is smaller (by $\sim 100$ residues) and without the kinase domain insert found in both the Pf PDPK and $T g$ PDPK. Notably, C. parvum does not have PKB (whereas P. falciparum does) or PKC (also not present in T. gondii or P. falciparum). C. parvum is like P. falciparum bearing 5 AGC protein kinases which is half of that found in $T$. gondii.

\section{CaMK group}

Despite the absence of $\mathrm{PKC}$, the prominence of CaMK family members indicates that regulation by calcium is clearly important in C. parvum parasites, as well as other apicomplexans [24]. Calmodulin ( $\mathrm{Cp} \mathrm{CaM}$, 
cgd2_810) with $85 \%$ sequence identity to human CaM and $4 \mathrm{CaM}$-like proteins (containing $<220$ residues and two to four EF-hands) including cgd2_3790, cgd2_1700, cgd3_3760, and cgd5_3920 were identified. From kinase domain homology, a prototypical CaMK enzyme may include cgd6_520 which was initially identified as a $C p$ CRK (CDPK-related kinase) [25]. Although it is $41 \%$ identical to and clusters with PfPK2 (a proven CaMK [26]) in the phylogenetic tree (Figure 2), the auto- inhibitory helix and CaM-binding site of this C. parvum kinase could not be readily identified. Cgd6_3400 clusters on a sister branch to the human CaMK enzymes and is $\sim 40 \%$ identical in sequence to them, but the auto-inhibitory helix and CaM-binding motif are not apparent in a sequence analysis. Cgd7_3890 (annotated as $C p C D P K 7$, but not containing EF-hands) contains motifs indicative of both the auto-inhibitory sequence and CaM-binding motif. Also, according to the

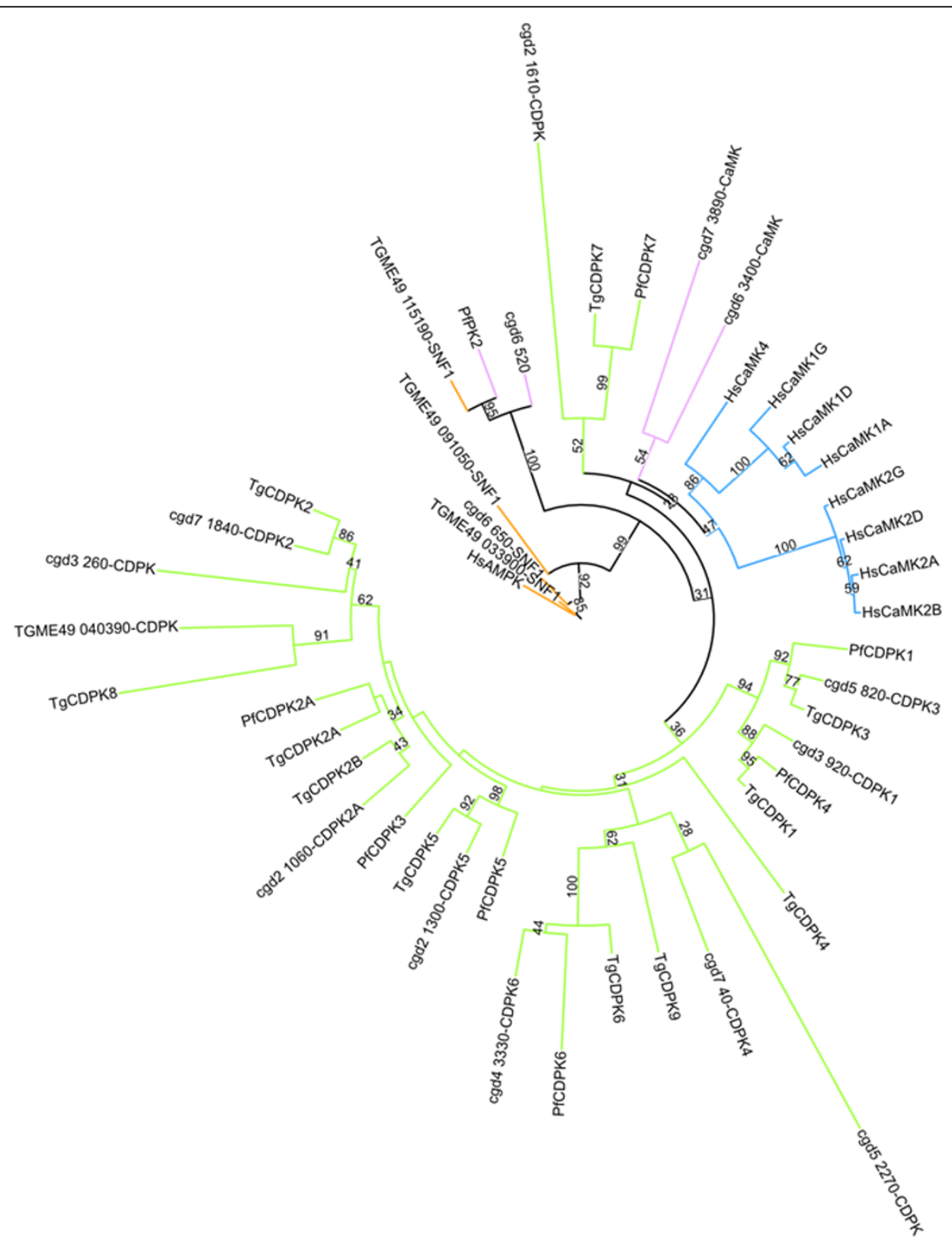

Figure 2 Maximum likelihood tree from a rapid bootstrap analysis for $C$. parvum CaMK family, also including select representatives of the CaMK families from human, P. falciparum, and $T$. gondii. The subfamilies are as follows: CDPK (green), CaMK (blue), SNF1/AMPK (orange), and potential apicomplexan CaM-binding kinases (pink). Note that although PFB0815w is annotated as CDPK1, it is the orthologue to TgCDPK3 (TGME40_105860) and PF07_0072 is annotated as PfCDPK4, but is actually the orthologue to TgCDPK1 (TGME49_101440). 
phylogenetic tree analysis, it is related to the human CaMK enzymes.

Like in plants and ciliates, the CDPK family dominates the apicomplexan CaMK group (Figure 2). Recently the mechanism of activation of CDPKs has been elucidated by our group through structural biology using full length structures of a few apicomplexan CDPK enzymes $[27,28]$. Many of the C. parvum CDPK enzymes have already been identified including: $C p$ CDPK1 (cgd3_920), С $p$ CDPK2 (cgd7_1840), С $p$ CDPK2A (cgd2_1060),
CpCDPK3 (cgd5_820), CpCDPK4 (cgd7_40), CpCDPK5 (cgd2_1300), and $C p C D P K 6$ (cgd4_3330) [24]. A previously unidentified member of this family (cgd3_260) has a kinase domain followed by 3 predicted EF hands (see Table 1 for the domain architecture of this and all of the CaMK enzymes discussed) and clusters amongst the other CDPK enzymes of C. parvum. Notably, the kinase domain of cgd3_260 is $>400$ residues containing a single large insert after the HRDxxxxN motif of subdomain VIB. According to OrthoMCL [29] this kinase

Table 1 Domain architecture of CaMK family members from C.parvum, T. gondii, and P. falciparum.

\begin{tabular}{|c|c|c|c|c|}
\hline Annotation & ID & Architecture & KD size & FL size \\
\hline CpCaMK & cgd6_520 & - -KD——- & 241 & 1103 \\
\hline PfPK2 & PFL1885 C & $-K D-$ & 253 & 509 \\
\hline TgSNF1 & TGME49_115190 & $-\mathrm{KD}-$ & 253 & 774 \\
\hline CpCaMK & cgd6_3400 & KD_-二-———- & 279 & 979 \\
\hline CpCaMK & cgd7_3890 & $\mathrm{KD}$ & 267 & 357 \\
\hline CpCDPK-like & cgd2_1610 & KD & 340 & 516 \\
\hline CpCDPK-like & cgd5_2270 & $-K D$ & 278 & 327 \\
\hline CpCDPK & cgd3_260 & KD——EF.EF & 426 & 1180 \\
\hline TgCDPK & TGME49_040390 & -—-KD-———————EF.EF & 562 & 2483 \\
\hline CpCDPK1 & cgd3_920 & KD.EF.EF.EF.EF & 256 & 538 \\
\hline PfCDPK1 & PFB0815w & KD.EF.EF.EF.EF & 269 & 524 \\
\hline $\operatorname{TgCDPK} 1$ & TGME49_101440 & KD.EF.EF.EF.EF & 332 & 582 \\
\hline CpCDPK2 & cgd7_1840 & CMB20.KD.EF.EF.EF.EF & 254 & 676 \\
\hline $\operatorname{TgCDPK} 2$ & TGME49_025490 & CMB20.KD.EF.EF.EF.EF & 254 & 711 \\
\hline CpCDPK2A & cgd2_1060 & -KD.EF.EF.EF.EF & 255 & 718 \\
\hline $\operatorname{TgCDPK} 2 \mathrm{~A}$ & TGME49_006590 & -KD.EF.EF.EF.EF.EF & 254 & 761 \\
\hline PfCDPK2B & PFF0520w & KD.EF.EF.EF & 254 & 509 \\
\hline $\operatorname{TgCDPK} 2 \mathrm{~B}$ & TGME49_042400 & -KD.EF.EF.EF.EF & 254 & 604 \\
\hline CpCDPK3 & cgd5_820 & KD.EF.EF.EF.EF & 256 & 523 \\
\hline PfCDPK3 & PFC0420w & -KD.EF.EF.EF.EF & 255 & 562 \\
\hline $\operatorname{TgCDPK} 3$ & TGME49_105860 & KD.EF.EF.EF.EF & 257 & 537 \\
\hline PfCDPK4 & PF07_0072 & KD.EF.EF.EF.EF & 258 & 528 \\
\hline TgCDPK4 & TGME49_037890 & ————KD.EF.EF.EF & 350 & 1171 \\
\hline CpCDPK4 & cgd7_40 & $-\mathrm{KD}(\mathrm{ZnF})$.EF.EF.EF- & 306 & 824 \\
\hline TgCDPK4A & TGME49_095760 & KD.EF.EF.EF & 266 & 534 \\
\hline CpCDPK5 & cgd2_1300 & -KD.EF.EF.EF.EF & 254 & 677 \\
\hline PfCDPK5 & PF13_0211 & -KD.EF.EF.EF.EF & 254 & 568 \\
\hline TgCDPK5 & TGME49_024950 & -KD.EF.EF.EF.EF & 254 & 681 \\
\hline CpCDPK6 & cgd4_3330 & EF.EF.KD.EF.EF.EF & 259 & 622 \\
\hline PfCDPK6 & PF11_0239 & -—————EF.EF.KD.EF.EF.EF & 252 & 1617 \\
\hline TgCDPK6 & TGME49_018720 & EF.EF.KD.EF.EF.EF.EF & 258 & 557 \\
\hline PfCDPK7 & PF11_0242 & EF.EF-——-_-—-—-_-——PH.KD- & 256 & 2265 \\
\hline $\operatorname{TgCDPK} 7$ & TGME49_028750 & -——- $\mathrm{PH}-\mathrm{KD}-—$ & 257 & 1346 \\
\hline $\operatorname{TgCDPK8}$ & TGME49_092060 & $-K D$ & 457 & 624 \\
\hline $\operatorname{TgCDPK9}$ & TGME49_017600 & $-E F . K D$ & 298 & 488 \\
\hline CpSNF1 & cgd6_650 & $\mathrm{KD}-二-—$ & 252 & 859 \\
\hline TgSNF1 & TGME49_033900 & KD- & 252 & 412 \\
\hline TgSNF1 & TGME49_091050 & - — - KD- & 252 & 827 \\
\hline
\end{tabular}

Each "-" within the architecture column represents up to 100 residues. Abbreviations include: FL for full length, KD for kinase domain, EF for EF-hands, ZnF for zinc finger, CMB20 for carbohydrate-binding module, and PD for Pleckstrin homology domain. 
has no known orthologues outside of Cryptosporidium spp. Also among the CDPK enzymes is cgd5_2270 (annotated as MELK (maternal embryonic leucine zipper kinase)), clustering with $C p C D P K 4$, but without EFhands and with small $N$ - and $C$ - terminal extensions of $\sim 25$ residues each. Clustered with the CDPK7 enzymes is cgd2_1610, which also does not have any EF-hands. Cgd6_650 is the CpSNF1 (homologous to the AMPmodulated protein kinases (AMPK)) according to its clustering on the CaMK phylogenetic tree presented herein (Figure 2) and its TgSNF1 orthologue (TGME49_033900). Upon comparison to P. falciparum with 13 CaMKs [17], there are 15 for C. parvum including the 8 CDPK enzymes with intact EF-hands, while $T$. gondii has 20 CaMKs listed with complete catalytic triads and 16 categorized as CDPKs [19].

\section{CK1 group}

Parasitic CK1 enzymes, including those from $P$. falciparum and $T$. gondii have attracted attention because of their unexpected binding to an immobilized cyclindependent protein kinase (CDK) inhibitor (purvalanol B) [30]. Two $C p C K 1$ enzymes have been identified herein, in comparison to 1 and 3 from $P$. falciparum and $T$. gondii, respectively $[17,19]$. Specifically, from $T$. gondii the cytosolic $T g C K 1 \alpha$ and the membrane bound $T g C K 1 \beta$ were isolated, while the third $T g C K 1$ (TGME49_047710) is uncharacterized. TgCK1 $\alpha$ could be selectively inhibited by purvalanol $\mathrm{B}$ and aminopurvalanol A over the host CK1 enzymes; and importantly, inhibition by aminopurvalanol (the more cell-permeable of the two) inhibits parasite cell growth [31]. All of these parasitic CK1 kinases share high sequence identity in the kinase domain (64\% to $89 \%$ ) and should be tested for similar inhibition profiles, including CDK inhibitors. Similar to $\operatorname{TgCK} 1 \beta$ which has a $C$-terminal tail implicated in membrane localization [31], both C. parvum enzymes have $C$-terminal tails indicating potential membrane localization.

\section{CMGC group}

There are 20 CMGC kinases in C. parvum which include the cyclin-dependent- (CDK), mitogen-activated(MAPK), glycogen-synthase- (GSK), and CDK-like (CDKL) kinases, as well as CK2 (casein kinase 2), CLK (CDC-like kinase), DYRK (dual-specificity tyrosine-(Y)phosphorylation regulated kinase), and SRPK (serine/ arginine-rich protein-specific kinase) [32]. In comparison, there are 20 CMGC kinases from $T$. gondii and 18 from $P$. falciparum $[17,19]$. Like those studied from $P$. falciparum and in other eukaryotic systems, a majority of CMGC kinases are involved in the control of cell proliferation and development, so their relative abundance in these organisms may reflect the variety of successive proliferative and non-proliferative stages which constitute their life cycles. CDK enzymes with the typical PSTAIRE cyclin-binding motif (where the terminal glutamate is the conserved residue in subdomain III forming a salt bridge with the catalyic lysine of subdomain II) include cgd3_1510 (which has no known orthologues outside Cryptosporidium spp.) and cgd5_2510 (orthologue of PfPK5 (MAL13P1.279) and TgPK2 (TGME49_018220)). Two other CDK enzymes were identified through their orthologues including cgd6_1420 with a SKTAIRE motif (orthologue to PFD0740w, CDC2-related protein kinase 3) and cgd7_430 with a HFTVLRE motif (orthologue to PF10_0141, MO15-related protein kinase (PfMRK) and TGME49_070330, CaMK). Note that "composite" kinases (i.e. those having characteristics from two different groups) have been previously characterized in apicomplexan parasites [33]. As well, there are cgd7_280 and cgd1_60 which have no known orthologues outside of Cryptosporidium spp., but are annotated as CDKs in CryptoDB, presumably due to the presence of PATSIRE and STTTLRE motifs. With respect to the first of 3 of the these $C p C D K$ enzymes with apicomplexan orthologues, the putative $C p$ MRK (cgd7_430) enzyme and its orthologue, $P f$ MRK (which has sequence homology to human $\mathrm{CDK}$ ) are the same size (320 and 324 residues, respectively) and share $50 \%$ sequence identity indicating that classes of compounds effective against PfMRK (of which there are several) should also be tried against CpMRK. These include the purines, quinolinones, oxindoles, and chalcones which have sub-micromolar $\mathrm{IC}_{50}$ values against the Plasmodium enzyme, but not the human CDK enzymes tested [34]. Also, PfMRK is inhibited by bromohydrosulfonylacetamides which possess moderate antimalarial activity against drug resistant parasites, but not broad spectrum CDK inhibitors [35]. PfMRK and its effector PfMAT1 (where the $C$-terminal domain of PfMAT1 is the PfMRK activator domain) have been demonstrated to be co-localized to the parasite nucleus and that PfMRK phosphorylates two plasmodial DNA replication proteins suggesting that PfMRK in the nucleus is involved with the regulation of the DNA replication machinery [36]. CDK enzyme, cgd6_1420 is half the size of its orthologue, PfCRK-3 (PFD0740w) with 1339 residues, which has been implicated as crucial in intraerythrocytic development of $P$. falciparum via regulation of gene expression [37]. Although PfCRK-3 bears an $N$-terminal domain of between 350-400 residues (depending on how the $>10$ inserts are counted), these enzymes still share $36 \%$ sequence identity. $C p$ CDK (cgd5_2510) and its orthologues are approximately the same size (at 295, 288, and 300 residues for C. parvum, P. falciparum, and T. gondii, respectively) and share from $65 \%$ to $72 \%$ sequence 
identity. Crystal structures of $C$. parvum CDK (cgd5_2510) have been solved with indirubin 3'-monoxime [PDB: 2QKR] and ADP [PDB: 3NIZ] bound by our group, as well as its orthologue structure (PfPK5) structure in the presence [PDB: 1V0P and 1V0O] and absence of inhibitors [PDB: 1OB3] by others [38].

C. parvum has 3 MAP kinases including cgd2_1960 (which is the orthologue of PF14_0294 (PfMAP-1)), cgd2_4340 (orthologue of PF11_0147 (PfMAP-2) and TGME49_007820), and cgd3_3030 (orthologue to TGME49_112570, TgMAPK-1). Each of the MAP-2 orthologues is similar in size from 508 to 563 residues, sharing from $49 \%$ to $55 \%$ sequence identity. Interestingly, like PfMAP-2, the C. parvum and T. gondii MAP2 orthologues share the peculiarity of not possessing the conserved TXY activation motif (just upstream of the conserved APE motif of subdomain VII) usually found in enzymes of this family [39]. Instead, each has a TSH at the same location; and furthermore no vertebrate MAPK enzymes deviate in this activation motif suggesting that the fine regulation mechanisms of these apicomplexan MAP-2 orthologues may present a collective opportunity for drug targeting. The remaining two $C$. parvum MAP kinases have the expected TxY motif, both in the form of TDY, like their respective $P$. falciparum and $T$. gondii orthologues. As well, using a reverse genetics approach, PAMAP-2 gene was shown to be essential for completion of the asexual cycle of $P$. falciparum, an unexpected result in view of the non-essentiality of the orthologous gene for $P$. berghei erythrocytic schizogony [40]. The PfMAP-1 and putative CpMAP-1 orthologues only share $38 \%$ identity, but both have substantial $C$-terminal extensions of 602 and 338 residues, respectively. The crystal structure of the kinase domain of putative CpMAP-1 [PDB: 3OZ6] has been solved by our group and is discussed below. Although cdg3_3030 and TgMAP- 2 share $42 \%$ sequence identity, they are notably distinct in size (overall, 566 versus 1212 residues, respectively) where the difference in size can be mostly attributed to the uncharacterized large $C$-terminal extensions of 247 and 794 residues, respectively.

Both the PfGSK-3 and CpGSK-3 (cgd4_240) bear an unusual $N$-terminal extension of about 70 residues (although there is no sequence conservation between the extensions and the catalytic domains are well-conserved). Notably, $C p$ GSK-3 has an insert between the catalytic lysine and just upstream of the gatekeeper motif. Its structure has been solved [PDB: 3EBO] by our group. Although the physiological functions of PfGSK-3 remain to be elucidated, a series of GSK-3 $\beta$ inhibitors tested on both PfGSK-3 and mammalian GSK-3 $\beta$ show a partially divergent sensitivity [41]. These results give promise to both PfGSK-3 and CpGSK-3 with respect to drug discovery.
Ten other members of the CMGC group were identified, including cgd8_3070 from its orthologue (TGME49_085160, a putative cyclin-dependent kinaselike 5). The putative $C p C K L$ and $T g C K L-5$ share $41 \%$ sequence identity; however, the $C$. parvum enzyme is significantly larger (551 residues versus 351 residues for $T g$ CKL-5) with $>10$ inserts relative to its $T$. gondii orthologue. In addition, a LAMMER kinase, 2 DYRK kinases, and a Skylp kinase were identified. Characterization of the PfLAMMER describes the enzyme as comprised of 2 domains, where the $N$-terminal domain is unique and containing multiple consensus phosphorylation sites, a number of RS/SR dipeptides, a large portion of charged residues, two putative nuclear localization signals, and 14 copies of a DKYD repeat and the $C$ terminal domain is typical of the LAMMER family [42]. By comparison, CpLAMMER (cgd5_250) has a smaller $N$-terminal domain comprised of $\sim 300$ residues (versus 550 for the PfLAMMER and 1330 for TgLAMMER), has a HTD motif (subdomain X), and is unusually rich in asparagine residues. The PfLAMMER is expressed specifically in the sexual stage; and thus the authors concluded that it might be important in the regulation of sexual differentiation [42]. C. parvum CMGC kinases belonging to DYRK subfamily include: cgd7_3050 bearing an HCD motif (orthologue of TgDYRK, TGME49_004280) and cgd8_5180 bearing a HAD motif (orthologue of PfDYRK, PF11_0156 and of another TgDYRK, TGME49_113180). These apicomplexan DYRK enzymes have low sequence identity between them and variable $N$-terminal domains ranging in size from almost 150 residues to over 700 residues. Cgd1_2960 is annotated as Sky1p-like and is implicated in RNA metabolism. The arginine of the HRD motif (subdomain $\mathrm{X}$ ) is not conserved and is replaced by threonine. Although it has a small $N$ - and $C$-terminal tails of 81 and 65 residues, respectively, it is the 4 inserts within the kinase domain that make this enzyme stand out, including one of almost 250 residues just upstream of the DFG motif of kinase subdomain VII. CK2 enzymes are the only family within the CMGC group that replaces the CMGC-arginine (found just upstream of the APE motif of subdomain VIII) with a lysine, as is observed herein for the $C$. parvum enzymes and their orthologues, cgd6_620 (orthologue of PF11_0096 CK2 $\alpha$ and TGME49_063070, CK2) and cgd7_1320, which has no known orthologues outside of Cryptosporidium spp. This CK2 specific motif may allow for the phosphorylation of substrates with proline or nonproline residues at the $\mathrm{P}+1$ position of the substrate due to its increased side-chain flexibility, giving $\mathrm{H}$-bonding to the main-chain oxygen at the strained position that the CMGC-arginine does, but also accommodating alterative $\mathrm{H}$-bonding [43]. The conserved CK2 
glycine following it may further contribute to the flexibility allowing a greater range of main-chain conformations, although cgd7_1320 does not conserve this glycine. The PfCK $2 \alpha$ has been characterized with protein kinase activity; and gene disruption experiments have shown that it is crucial to the asexual blood stage in Plasmodium, thereby providing evidence that it may be a drug target [44]. Furthermore, it has differential susceptibility to a small molecular inhibitor making it an attractive target for antimalarial intervention, and potentially its orthologues in other parasitic diseases. Finally, 3 uncharacterized CMGC members with no known orthologues outside of Cryptosporidium spp. were also identified: cgd1_810 (which has a > 600 residue $N$-terminal extension and > 600 residue kinase domain), cgd7_4850 (which is relatively small at 286 residues, has an asparagine instead of an arginine in the HRD motif), and cgd2_3890.

\section{Atypical group}

Atypical protein kinases (aPKs) lack sequence similarity to the eukaryotic protein kinase domain hidden Markov model profile and as such are unrelated (or only distantly related) by sequence to ePKs; however, they have been shown experimentally to have protein kinase activity or are clear homologues of aPKs with demonstrated protein kinase activity. There are four C. parvum kinases that are identified as atypical based on their $P$. falciparum and T. gondii orthologues, including 2 RIOs (cgd7_440 and cgd5_2000) and 2 ABC1s (cgd8_3250 and cgd5_2400) (Additional File 1, Table S1). There may be as many as 24 from $T$. gondii [19] and 4 from $P$. falciparum [17] (including the RIOs and $\mathrm{ABC} 1 \mathrm{~s}$ ).

\section{TKL group}

In $C$. parvum, there are 3 TKL enzymes including cgd8_2430, cgd3_2900, and cgd3_4310 with each having $P$. falciparum and/or T. gondii TKL orthologues, while $T$. gondii and $P$. falciparum each contains 5 TKL enzymes (Additional File 1, Table S1). Recently, work on PfTKL-3 (PF13_0258, the orthologue of cgd3_4310 (the putative $C p$ TKL-3) has demonstrated that it is essential for asexual parasite proliferation in human erythrocytes [45]. Furthermore, the authors showed that it undergoes in vitro autophosphorylation and phosphorylates exogenous substrates both of which are dependent on the presence of a sterile $\alpha$-motif (SAM) domain at the $N$-terminus. Although these C. parvum and P. falciparum TKL-3 orthologues only share $30 \%$ overall sequence identity, they both have a SAM domain, as well as putative MORN (membrane occupation and recognition nexus) motifs that are $N$-terminal to the kinase domain and not shared by the TgTKL-3 orthologue. In the case of the $P$. falciparum orthologue, there is an additional $N$-terminal domain of $>1300$ residues (bearing PEXEL and 14_3_3 mode II binding motifs) upstream of the SAM domain, correspondingly this domain is only $\sim 300$ residues in the C. parvum enzyme. Although CpTKL-1 and PfTKL-1 (cgd8_2430 and PFB0520w, respectively) also bear these SAM and MORN motifs, the third CpTKL does not (cgd3_4310).

\section{OPK group}

There are 2 clades of protein kinases entirely unique to Apicomplexa, namely rhoptry kinases ( $T$. gondii and Neospora caninum only) and FIKK kinases (found only in Plasmodium). We conducted BLAST analysis of the sequences of all known Toxoplasma ROP kinases against the $C$. parvum genome and did not find a single orthologue. However, amongst a clad of others, a FIKKcontaining kinase was identified (cgd5_4390) which has been previously annotated as a R45-like orthologue (and named $C p R 45)$ by Schneider et al. from a tBLASTn search using the protein kinase domain of PFD1175w [46]. This protein is phylogenetically close to the Plasmodium FIKK cluster and also $40 \%$ identical in sequence to PfFIKK8 (MAL8P1.203), with both lacking the PEXEL motif found in most other $P$. falciparum FIKK kinases. It is also the orthologue of a catalytically incomplete TgFIKK kinase (TGME49_089050). Although there are clades of OPK enzymes amongst the annotated protein kinases of $C$. parvum, unique conserved motifs potentially identifying a unique Cryptosporidium class of protein kinases are not readily apparent. Since $C p \mathrm{OPK}$ enzymes comprise almost $35 \%$ of the kinome, we have divided them into their respective clades to simplify the discussion and are not implying a further organization of the C. parvum kinome at this point. OPK clade 1 is comprised of 4 protein kinases including: cgd2_3190 (with a $>300$ residue $N$-terminal extension) which is the orthologue of a putatively identified AGC-related PFC0385 c from P. falciparum and the T. gondii aurora kinase TGME49_003010; cgd6_5060 which contains $N$ and $C$-terminal extensions of $\sim 200$ residues and has a $P$. falciparum orthologue that is also AGC-related; cgd6_5240 which has no known orthologues outside of Cryptosporidium spp.; and cgd3_3180. OPK clade 2 includes 6 enzymes: cgd7_2000 which has no known orthologues outside of Cryptosporidium spp. and $N$ - and $C$-terminal extensions of 436 and 72 residues; $C p R 45$ (already discussed); cgd7_1190 which is an orthologue of PFC0485w (CaMK) and TGME49_018550 (PIK3R4 kinase-related protein); cgd1_3230 which has no known orthologues and a $C$-terminal extension that is $>500$ residues; cgd6_4960; and cgd8_1660. OPK clade 3 contains 6 kinases that very little is known about including: cgd4_990 and cgd4_1610, both with no known orthologues outside of Cryptosporidium spp; cgd8_5120 which 
may be related to the CMGC kinases based on the annotations of its orthologues; cgd7_1330 which may contain an $N$-terminal transmembrane domain and has green plant/algae orthologues; cgd7_3430 which has a TKL orthologue from $T$. gondii, and cgd2_2310 with uncharacterized apicomplexan orthologues. OPK clade 4 contains cgd1_1490 (with a $>250$ residue $C$-terminal domain) which is an orthologue to PFL1370w (NIMA related protein kinase 1 ) that also has a $C$-terminal extension (> 750 residues). PfNEK-1 is able to autophosphorylate and phosphorylate select protein substrates, including PfMAP-2 [47]. There is also cgd1_890 which has a kinase domain with a large insert making it more than twice the size (at 659 residues) of a typical kinase domain. It is the orthologue to PFF1370w (PfPK4) and TGME49_029630 (kinase with an incomplete catalytic triad). Cgd3_3230 has substantial $N$ - and $C$-terminal extensions (284 and 920 residues, respectively) and is the orthologue to PF14_0423 (eukaryotic initiation factor 2 alpha kinase 1). Cgd7_3760 is an orthologue to NEK kinases from $P$. falciparum and $T$. gondii. Cgd7_5050 is annotated as NIMA related kinase 5 and contains an $\mathrm{N}$-terminal domain $>1000$ residues. Interestingly, cgd4_3710 has an unusually large (> 400 residues) kinase domain (typically $\sim 255$ residues) that is a function of 4 inserts including one after the catalytic lysine (from subdomain II), another after the HRDxxxxN motif of subdomain VIB, one after the APE motif of subdomain VIII, and the last after the conserved aspartic acid of subdomain IX. The remaining 3 kinases include cgd7_3080 which has a T. gondii Wee kinase orthologue, cgd8_1230, and cgd8_2180.

\section{Activity of $C p C D P K 1, C p C D P K 2, C p C D P K 3$, and $C p C D P K 4$}

The effect of calcium on the activity of constructs containing the kinase domain $(\mathrm{KD})$ and $\mathrm{CAD}(\mathrm{CDPK}$ activation domain) of $C p \mathrm{CDPK} 1, C p \mathrm{CDPK} 2, C \bar{p} \mathrm{CDPK} \overline{3}$, and $C p \mathrm{CDPK} 4$ was tested using the pyruvate kinase-lactate dehydrogenase (PK-LDH) assay and the peptide substrate Syntide-2 (a common CaMK substrate with the sequence PLARTLSVAGLPGKK). They exhibited a range of phosphorylation activities (Table 2), but all showed an increase in activity corresponding to an increase in calcium concentrations with turnover numbers of $11 \pm 1,9$ $\pm 2,64 \pm 6$, and $3 \pm 1 \mathrm{mM}^{-1} \mathrm{~min}^{-1}$, respectively, and in the range of values previously determined for other CDPKs with the exception of $C p C D P K 3$ [28]. The difference in the catalytic efficiencies of these four CDPKs is on the order of 300-fold for the same common kinase peptide substrate, Syntide-2. Indeed, the CDPK enzymes are expected to have different substrate specificities. For example $P f C D P K 1$ is capable of phosphorylating myelin basic protein (MBP), histone 1 , and casein, while PfCDPK2 only recognizes MBP as a substrate [48].
Table 2 Kinetics of CpCDPK1 (cgd3_920, true FL version), CpCDPK2 (cgd7_1840), CpCDPK3 (cgd5_820), and CpCDPK4 (cgd7_40).

\begin{tabular}{lcccc}
\hline Enzyme & $\begin{array}{c}\mathbf{K}_{\mathbf{M}} \text { syntide } \\
(\boldsymbol{\mu M})\end{array}$ & $\begin{array}{c}\mathbf{K}_{\mathbf{M}} \mathbf{A T P} \\
(\boldsymbol{\mu M})\end{array}$ & $\begin{array}{c}\mathbf{k}_{\mathbf{c a t}} \\
\left(\mathbf{m i n}^{-1}\right)\end{array}$ & $\begin{array}{c}\mathbf{k}_{\mathbf{c a t}} / \mathbf{K}_{\mathbf{M}} \\
\left(\mathbf{m M}^{-1} \cdot \mathbf{m i n}^{-1}\right)\end{array}$ \\
\hline CpCDPK1 & $157 \pm 17$ & $20 \pm 3$ & $1370 \pm 40$ & 8726 \\
CpCDPK2 & $260 \pm 13$ & $22 \pm 1$ & $240 \pm 5$ & 923 \\
CpCDPK3 & $156 \pm 69$ & $81 \pm 4$ & $367 \pm 37$ & 2353 \\
CpCDPK4 & $426 \pm 47$ & $29 \pm 8$ & $12 \pm 1$ & 28 \\
\hline
\end{tabular}

\section{Effect of the $\mathrm{N}$-terminal latch on CDPK activity}

The $C p C D P K 1$ construct tested herein has a complete $N$-terminal domain comprising 55 additional residues over the other three CDPK enzymes tested, where these constructs expressed do not have this $N$-terminal domain. The CDPK $N$-terminal domain has been postulated to function as a structural latch that will enable full kinase activity to be maintained once calcium has been depleted. Our data on $C p C D P K 1$ in the presence of calcium shows that there is little difference between the activity of a construct with an intact latch versus a construct without a latch, a difference of $\sim 50$ residues upstream of the subdomain 1 GxGxxG motif (data not shown). However there is some sequence conservation of hydrophobic residues just upstream of the GxGxxG motif for P. falciparum, T. gondii, and C. parvum; and this may indicate a conserved latch regulatory mechanism for apicomplexan CDPKs [28]. Specifically, hydrophobic patterns including a PGMF motif in at least 6 apicomplexan CDPKs and a FxRxxFILxxxG (or the variation of FxRxxooxxxxL), where $x$ is any residue and $o$ is a hydrophobic residue, in 17 CDPKs which may signify that the application of such a regulatory mechanism reliant on the interaction of hydrophobic residues is used by apicomplexan CDPKs (Additional File 2, Table S4).

\section{Inhibition of $C p C D P K 1$ by pyrazolopyrimidine derivatives} Inhibition of $C p C D P K 1$ was thoroughly investigated by screening with a series of compounds designed to exploit the small gatekeeper that is naturally occurring in $C p C D P K 1$, but not in the other $C p C D P K s$ (Additional File 3, Table S5). For example, similar to previous results [13], pyrazolopyrimidine derivatives are ineffective against kinases with bulky gatekeepers (found just downstream of the catalytic lysine in a Yoo*xooxGGELFxxI motif, where o is hydrophobic, $\mathrm{x}$ is any, and * is the gatekeeper), but $C p C D P K 1$ is expected to be sensitive to such inhibitors owing to the presence of a glycine instead of the typical methionine present in the remaining $C p C D P K s$. Accordingly, over a dozen PP1 derivatives were found that inhibit $C p C D P K 1$ with $\mathrm{IC}_{50}$ 's less than $10 \mathrm{nM}$ (Table 3). 
Table 3 IC $_{50}$ values for CpCDPK1 inhibitors tested.

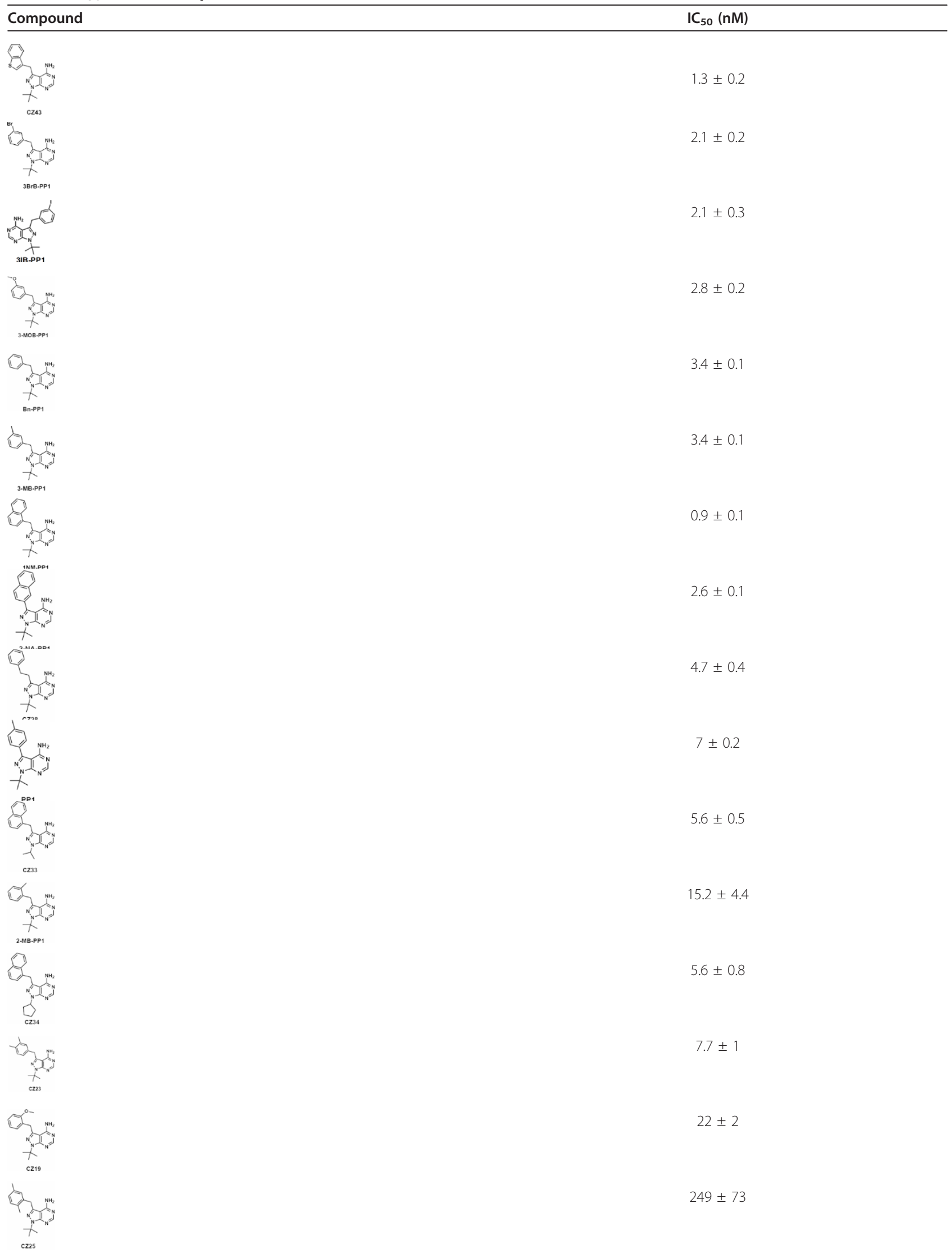




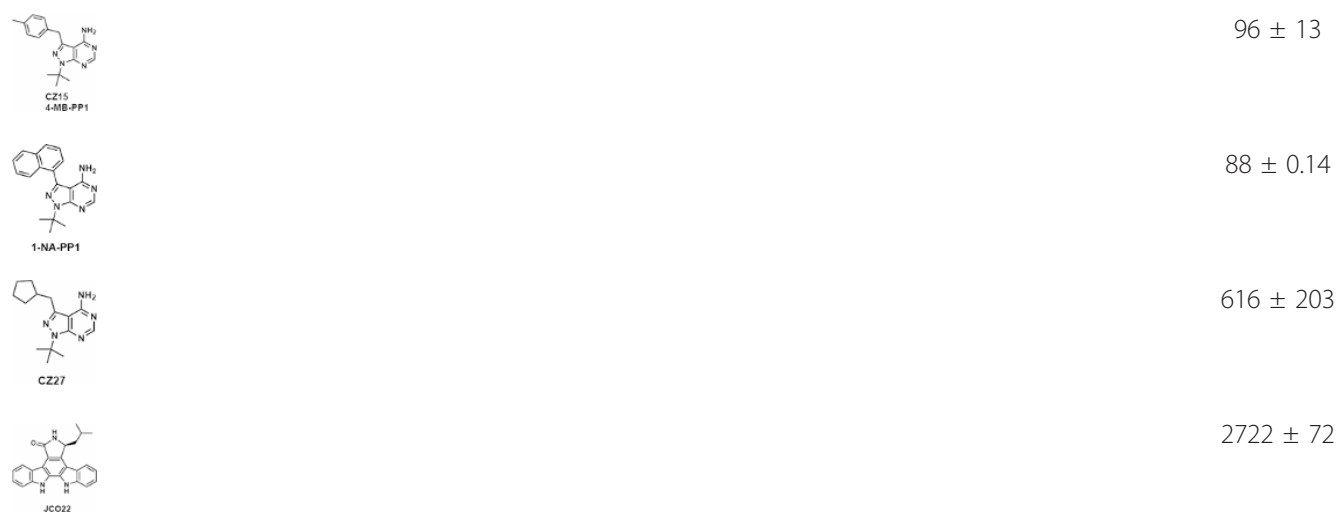

All compounds were tested using $500 \mu \mathrm{M}$ ATP and $500 \mu \mathrm{M}$ Syntide2 as described in Methods. A concentration range from $1 \mathrm{nM}$ to $1 \mu \mathrm{M}$ for all compounds was used except for CZ25, CZ15 and 1nA-PP1 (2 nM to $2 \mu \mathrm{M}), \mathrm{CZ27}(4 \mathrm{nM}$ to $4 \mu \mathrm{M})$ and JC022 (8 nM to $8 \mu \mathrm{M})$.

\section{Structural characterization of select $C p C D P K s$}

The CDPKs have a range of different type of domain organizations, as shown in Table 1. To date, C. parvum and $T$. gondii CDPK structures solved include KD, CAD (including 4-EF hands), and intact KD-CAD (with and without calcium bound). Herein, we present the solved KD structures from $C p C D P K 1, C p C D P K 2$, and CpCDPK4 (Figure 3 and Table 4).

\section{CpCDPK1 and $C p C D P K 3$ structures}

The structures of $C p$ CDPK1 [PDB: 3IGO] and $C p$ CDPK3 [PDB: 3LIJ] with both the KD and CAD domains intact have been solved by our group. These and other CDPK structures including calcium-free and calcium-bound forms have been used to describe a model for the activation of the CDPK family of enzymes and to characterize the CAD domain, a novel member of the EF-hand containing family, whose structure has also been solved for $C p$ CDPK3 [PDB: 3L19] [27,28]. The exploitation of the $C p C D P K 1$ ATP-binding site featuring a glycine-gatekeeper has also been described in detail including its corresponding full length kinase structure with inhibitors bound [PDB: 3NCG and 3MWU] [13]. Herein, we have solved the KD structure of $C p$ CDPK1 [PDB: 3DFA] in apo form as well as with a PP1-derivative (1-tert-butyl-3(3-methylbenzyl)-1H-pyrazolo[3,4-d]pyrimidin-4-amine, 3MB-PP1) bound [PDB: 2WEI]. The overall fold of the KD structures with and without the PP1 derivative bound are similar (Figure 4A). In both structures, the glycine-rich loop is clamped down reflective of the activated form, the tip of which (containing Phe87, all numbering from full length and same as for PDB: 2WEI) is tucked into a pocket created by the crossing of $\beta$-sheet 3 and the residues just prior to the activation loop. The activation loop, which moves closer to the active site in the 3MB-
PP1 structure sits lower and retains an additional turn at the top of the $\alpha$-helix $G$ in the apo structure. The pyrazolo-pyrimidine in the PP1 molecule occupies the same space where the adenine in ATP is typically found, forming $\mathrm{H}$-bonds with the backbone of Glu153 and Tyr155 (Figure 4B). N8 of the 3MB-PP1 is linked by a water to the activating Lys105. The large, hydrophobic methylbenzyl group of 3MB-PP1 sits deep within the pocket lined by Leu222, Leu138, Ile150, and Met136. In most other kinases, this pocket is ablated by the side-chain of a large gatekeeper residue.

\section{$C p C D P K 2$ structure}

$C p$ CDPK2 comprises an $N$-terminal domain that is predicted to bind a carbohydrate, KD, and the CAD. We have solved the crystal structures of CpCDPK2 KD with indirubin E804 (3-(\{[(3S)-3,4-dihydroxybutyl $]$ oxy $\}$ amino)-1H,2'H-2,3'-biindol-2'-one) bound [PDB: 3F3Z], as well as the apo form [PDB: 2QG5]. Both structures have completely ordered activation loops, with $\alpha$-helix $C$ of the indirubin-bound structure not completely in the activated form (Figure 5). There is a dramatic difference in glycine-rich loops between the apo and the indirubinbound structures. In the apo form, the loop is moved up and away from the activation site, adopting a conformation less amenable to binding ATP. With the indirubin-bound structure, the backbone atoms from the loop move up to $8 \AA$ closer to the active site. Once again, the indole moiety is interacting with the backbone hinge residues Glu103 and Cys105 (numbering is identical in both structures, but 194 less than full length numbering). The hydroxyl groups from the "tail" of the indirubin form a series of $\mathrm{H}$-bonds with residues from the $\mathrm{C}$ lobe of the catalytic domain. Glu109 is pulled up by this interaction, such that the $\alpha$-helix D is more ordered and 


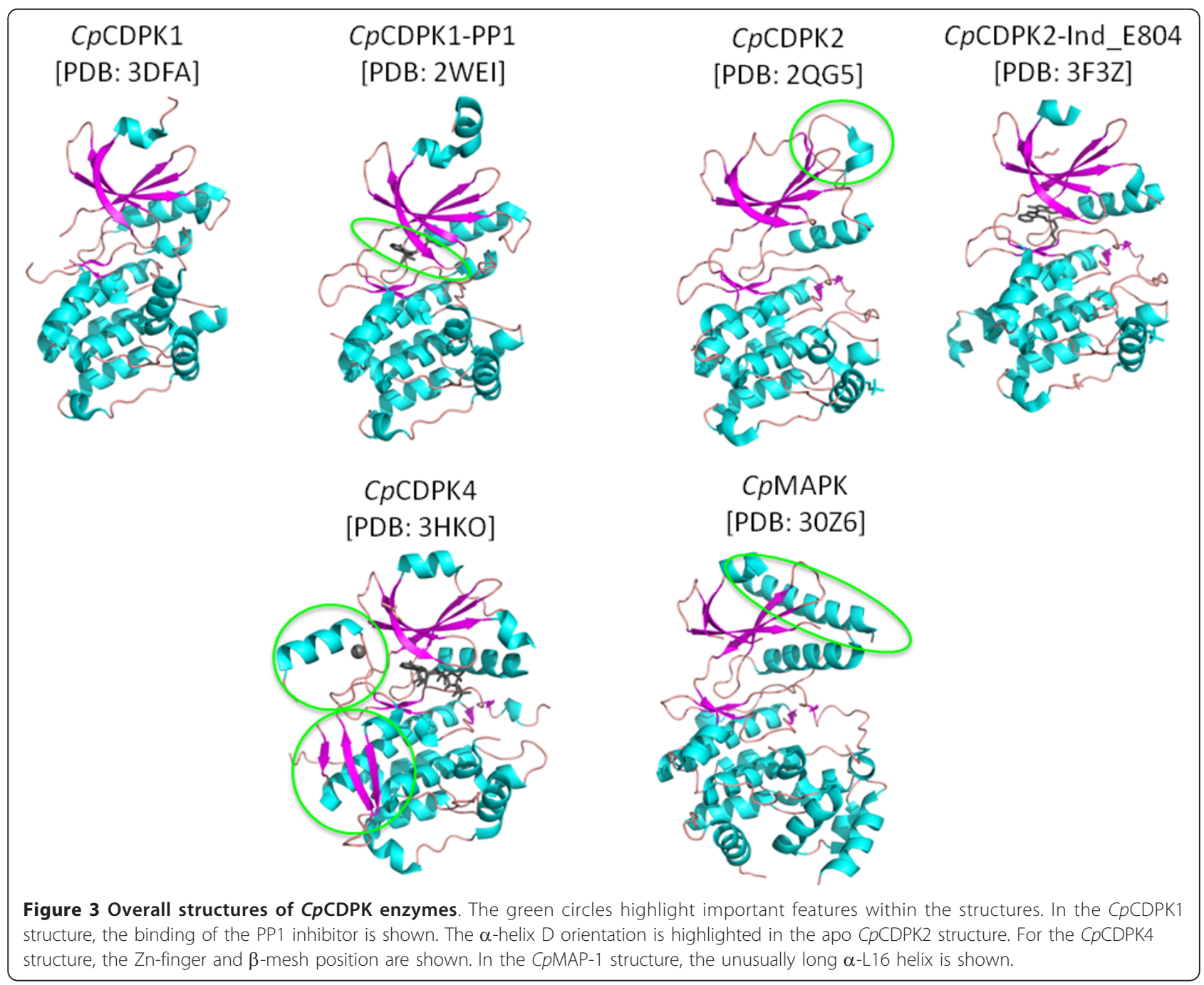

contains an additional turn compared to the $\alpha$-helix D from the apo structure.

\section{CpCDPK4 structure}

The KD structure of $C p C D P K 4$ has been solved in the presence of a non-hydrolyzable ATP analogue (phosphoaminophosphonic acid-adenylate ester) [PDB: $3 \mathrm{HKO}$. Sequence alignment shows that $C p C D P K 4$ has a unique insert within the kinase domain that is particularly cysteine-rich in the centre: ${ }^{101}$ LNVFIDDSTGKCAMDVVKTQICPCPECENEEAINGSIHGFRES ${ }^{140}$ (with all numbering from $\mathrm{PDB}$ : $3 \mathrm{HKO}$ which is 125 less than full length numbering). This insert is situated between the hinge region and $\alpha$-helix F. It consists of an antiparallel $\beta$-mesh that interacts with the helical $C$-lobe of the KD and a helix that interacts with the $N$-terminal $\beta$ lobe (Figure 6A). Embedded in the helix is a zinc ion coordinated by His93, Cys122, Cys124 and Cys127 (Figure $6 \mathrm{~B}$ ). This zinc finger is not present in any other known protein $K D$, based on searches by sequence and structure (also from personal communications with Gerald Manning and Stefan Knapp), making the insert particularly interesting and unique.

When $C p C D P K 4$ is overlaid with the structure of PKC-I [PDB: 3A8W] that includes the C-terminal residues, we see that the zinc finger lies within the same vicinity as the $C$-terminal tail of PKC-ı (Figure 6D). This tail is believed to bring a hydrophobic residue, Phe543 into van der Waals contact with the adenosine head of ATP and the tail forms $\mathrm{H}$-bonds with the glycine-rich loop, thereby ensuring an activated state for PKC-ı [49]. The zinc finger of $C P C D P K 4$ is in the vicinity of the $N$ lobe and the exact volume occupied by the PKC-ı $C$ terminal tail. Instead of a phenylalanine, however, an isoleucine (Ile121) is in position to maintain the more hydrophobic area for the adenosine head group. In addition, for human CLK1 and CLK3 (PDB: 1X57 and 2EU9, respectively), the $C$-terminal lobe bears an 
Table 4 Data collection, phasing, and refinement statistics for the C. parvum CDPK and MAP-1 structures.

\begin{tabular}{|c|c|c|c|c|}
\hline Structure & & CpCDPK1 & CpCDPK1-PP1 & CpCDPK2 \\
\hline PDB Code & & 3DFA & 2WEI & 2QG5 \\
\hline Space Group & & P43212 & P43212 & $\mathrm{C} 2$ \\
\hline \multicolumn{5}{|l|}{ Cell Dimensions } \\
\hline & a $(\AA)$ & 69.45 & 68.9 & 138.18 \\
\hline & $b(\AA)$ & 69.45 & 68.9 & 86.48 \\
\hline & $c(\AA)$ & 137.41 & 130.46 & 87.96 \\
\hline & $A\left({ }^{\circ}\right)$ & 90 & 90 & 90 \\
\hline & $\beta\left(^{\circ}\right)$ & 90 & 90 & 96.37 \\
\hline & $\gamma\left({ }^{\circ}\right)$ & 90 & 90 & 90 \\
\hline Wavelength & & 0.91996 & 0.99989 & 1 \\
\hline Resolution $(\AA ̊)$ & & $50-2.45(2.54)$ & $33-1.65(1.74)$ & $50-2.3(2.36)$ \\
\hline Unique reflections & & & 38607 & 38421 \\
\hline$R_{\text {merge }}$ & & $.072(.471)$ & $.11(.70)$ & $.11(.45)$ \\
\hline$|/ \sigma|$ & & $11.2(4.53)$ & $10.2(2.3)$ & $12.4(1.5)$ \\
\hline Completeness (\%) & & $96.9(83.5)$ & $99.9(100)$ & $88.4(60.7)$ \\
\hline Redundancy & & $13.2(11.1)$ & $8.3(7)$ & $3.4(2.0)$ \\
\hline \multicolumn{5}{|l|}{ Refinement } \\
\hline Resolution & & $\begin{array}{c}50-2.45 \\
(2.52)\end{array}$ & $33-1.65$ (1.693) & $40-2.3(2.36)$ \\
\hline Number of Reflections & & 12590 & 36676 & 38421 \\
\hline Test Set & & 611 & 1837 & 2104 \\
\hline$R_{\text {work }} / R_{\text {free }}$ & & $.234 / .263$ & $.197 / .229$ & $.224 / .286$ \\
\hline Number of Atoms & & 2183 & 2486 & 7227 \\
\hline Mean $B_{\text {factor }}$ & & 59.4 & 22.1 & 38.03 \\
\hline Ramachandran Favored & & 96.1 & 97.5 & 95.8 \\
\hline Ramachandran Disallowed & & 0 & 0 & 0 \\
\hline \multicolumn{5}{|l|}{ RMS deviations } \\
\hline & Bond length $(\AA)$ & 0.007 & 0.014 & 0.013 \\
\hline & Bond angle $\left(^{\circ}\right)$ & 1.014 & 1.41 & 1.351 \\
\hline Structure & & CpCDPK2-Ind-E804 & CpCDPK4 & CpMAP-1 \\
\hline PDB Code & & $3 F 3 Z$ & $3 \mathrm{HKO}$ & $30 Z 6$ \\
\hline Space Group & & $\mathrm{C} 2$ & P212121 & P212121 \\
\hline \multicolumn{5}{|l|}{ Cell Dimensions } \\
\hline & a $(\AA)$ & 63.32 & 54.48 & 74.30 \\
\hline & $b(\AA)$ & 82.86 & 63.04 & 96.75 \\
\hline & $c(\AA)$ & 62.15 & 84.04 & 119.99 \\
\hline & $\mathrm{A}\left({ }^{\circ}\right)$ & 90 & 90 & 90 \\
\hline & $\beta\left(^{\circ}\right)$ & 111.61 & 90 & 90 \\
\hline & $\gamma\left({ }^{\circ}\right)$ & 90 & 90 & 90 \\
\hline Wavelength & & 1.5418 & 0.978 & 0.97924 \\
\hline Resolution $(\AA)$ & & $50-1.84(1.91)$ & $50-1.80(1.86)$ & $50-2.4(2.44)$ \\
\hline Measured reflections & & & & 607387 \\
\hline Unique reflections & & & 27359 & 62534 \\
\hline$R_{\text {merge }}$ & & $0.054(.606)$ & $0.073(.555)$ & $0.087(.921)$ \\
\hline$|/ \sigma|$ & & $x(2.5)$ & $20.5(1.53)$ & $14.8(1.34)$ \\
\hline Completeness (\%) & & $99.2(95.7)$ & $99.2(92.4)$ & $99.2(98.7)$ \\
\hline Redundancy & & $3.7(3.5)$ & $6.1(2.50)$ & $3.8(3.8)$ \\
\hline
\end{tabular}


Table 4 Data collection, phasing, and refinement statistics for the C. parvum CDPK and MAP-1 structures. (Continued)

\begin{tabular}{rccc}
\hline Refinement & & & \\
\hline Resolution & $50-1.84(1.89)$ & $50-1.8(1.84)$ & $45-2.4(2.44)$ \\
\hline Test Set & 25653 & 27292 & 33427 \\
\hline$R_{\text {work }} / R_{\text {free }}$ & 1303 & 1376 & $.232 / .259$ \\
\hline Number of Reflections & $.189 / .230$ & $.204 / .246$ & 5390 \\
\hline Mean B Bactor & 2462 & 2789 & 51.1 \\
\hline Ramachandran Favored & 27.47 & 97.2 & 0 \\
\hline Ramachandran Disallowed & 98.2 & 0 & 0 \\
\hline & Bond length $(\AA)$ & 0 & 0.7 \\
\hline
\end{tabular}

insertion between the two sheets $\beta 7$ and $\beta 8$, termed the CLK-specific $\beta$ hp- $\beta$ hp' hairpin, and renders the substrate docking groove inaccessible (as compared to the substrates of MAPK which are able to access a similar groove) [50]. Although the CLK-specific hairpin partially overlaps with the $\beta$-mesh portion of the $C p$ CDPK 4 insert, it is distinct in orientation and lacks sequence identity to the $C p C D P K 4$ insert (Figure 6E). Thirdly, although using a deletion mutant to obtain the crystal structure of human SRPK1 that truncates the $N$-terminus by 41 residues and eliminates a spacer of 217 residues (residues 256-473), there is some overlap between the location of the remaining spacer and the $C p C D P K 4$ insert (Figure 6F). There are two small motifs that share sequence identity between the $C p C D P K 4$ insert and SPRK, specifically ${ }^{119} \mathrm{TxIxxxP}^{125}$ and ${ }^{128} \mathrm{NExxI}^{132}$ (numbering from PDB: $3 \mathrm{HKO}$ ), but only the latter shares spatial and structural similarity, forming the first $\alpha$-helix in each insert structure but in a nearly orthogonal orientation. For SPRK, the two spacer inserts identified in the structure are necessary for maintaining an active conformation [51]. Although these other inserts have been identified, only further research will determine what particular role both the zinc finger and $\beta$-mesh play in the regulation of $C p C D P K 4$. However, the location and other aspects of the $\beta$-mesh suggest that it may affect

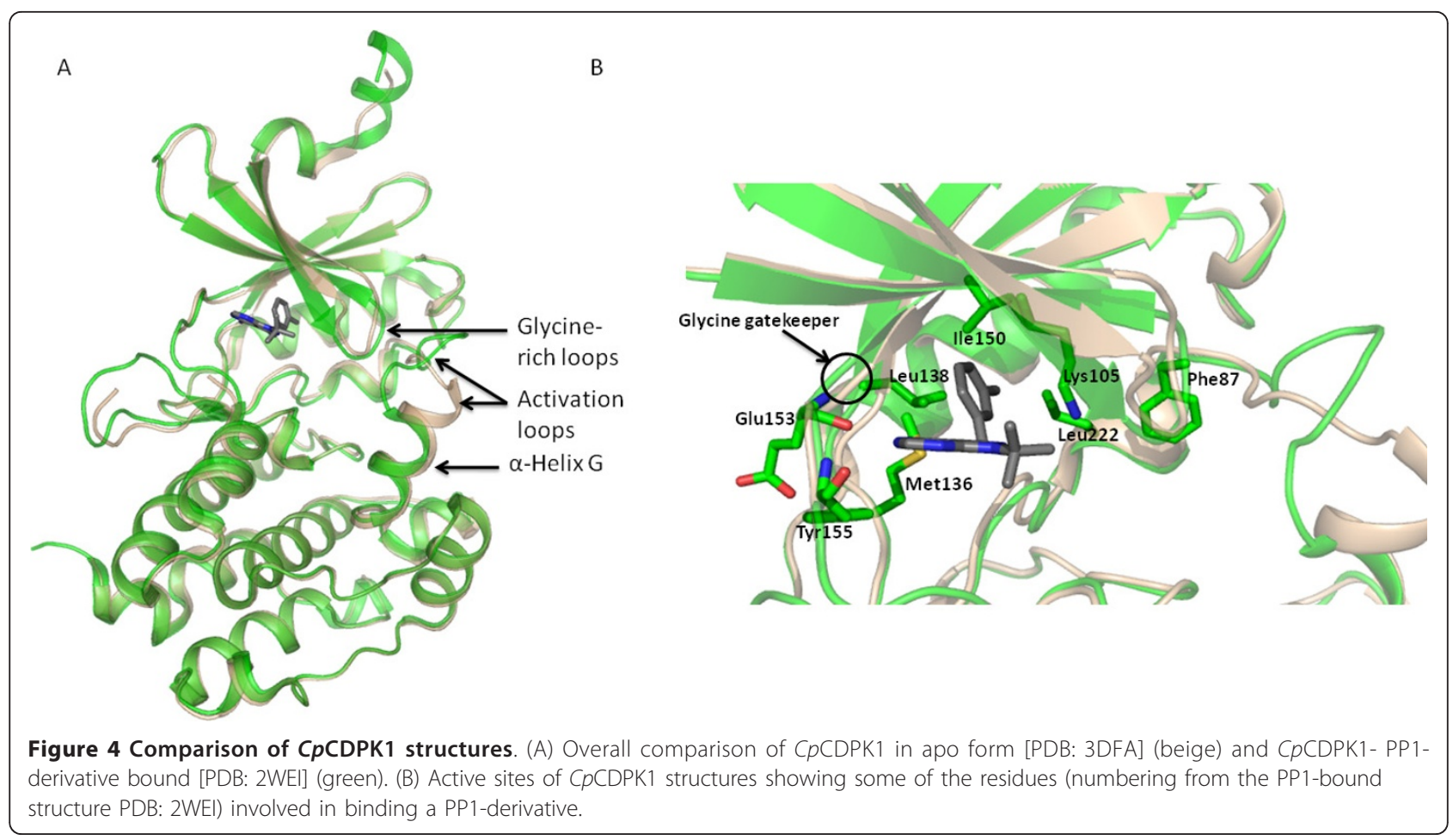




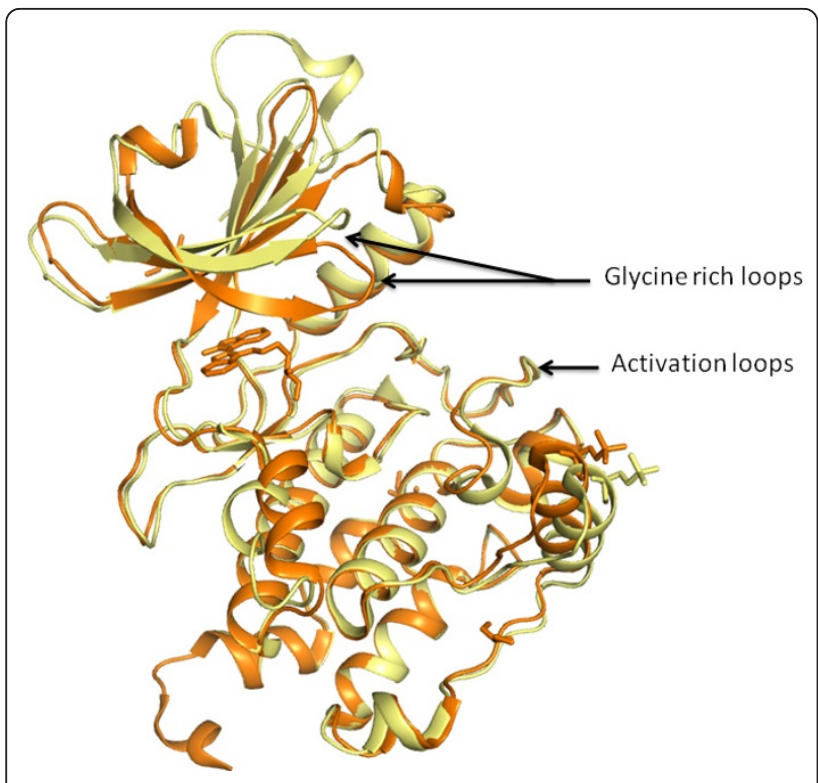

Figure 5 Comparison of CpCDPK2 structures. CpCDPK2 in apo form [PDB: 2QG5] (yellow) and CpCDPK2- indirubin E804 bound [PDB: 3F3Z] (orange) shows the shift in the glycine-rich loop.

the activation state of this kinase in a specific way. To illustrate, the $\beta$-mesh formed downstream of the zinc finger is indeed novel in that its $C$-terminal side forms part of the $\alpha$-helix D. This helix consists of less than two turns, and at the head features a histidine (His96) that is in H-bonding distance with the alcohol groups of the ribose ring. This position is typically taken up by a glutamate in catalytically active kinases. Additionally, when overlaid with the inactive form of a more prototypical CDPK with the CAD domain ( $T g C D P K 1$ [PDB:3KU2]), we can see that the bottom of this $\beta$ mesh would interfere sterically with the inhibitory $\mathrm{CH} 1$ helix of the CDPK (Figure 6C). All in all, this suggests that the zinc-bound form of this particular CDPK could be constitutively active, as a reversion to the inactive state would be blocked. Attempts to find structures, either active or inactive of the $C p C D P K 4$ with CAD domain intact are underway.

\section{CpMAP-1 structure}

The CpMAP-1 structure [PDB: 3OZ6] was solved in the absence of any ATP mimic or inhibitor, and as such the glycine-rich loop, which interacts with $\beta$ - and $\gamma$-phosphates of the ATP, is disordered at the tip in our structure (Figure 7 ). In comparison with a prototypical MAPK, such as ERK2, the MAPK-specific helix L16 is longer in our structure, and forms more hydrophobic interactions with the $\alpha$-helix C. The activation loop is moved out of position and no phosphorylation states have been found in the electron density. As is typical with structures lacking ATP/mimic, a good portion of the activation loop is disordered with residues from 175 to 189 absent.

\section{Conclusions}

Overall, protozoan kinases are appealing drug targets, as many are already known to be involved in essential cell cycle regulation; and there is an enormous availability of inhibitors from the extensive work done on human kinase targets. There are several examples of specific kinases to be considered for further investigation with respect to their potential in C. parvum drug design, as their orthologues in Plasmodium and Toxoplasma perform critical functions (see Additional File 1, Table S1 for C. parvum orthologues). For example, PfPKG is essential in the blood stage and in gametogenesis of $P$. falciparum infection [22,23], as well, TgPKG is the likely target of an anti-toxoplasmosis compound [52]. PfMRK and PfPK5, respectively, are inhibited by compounds that also modestly inhibit P. falciparum growth [34]. TgCK $1 \alpha$ is inhibited by aminopurvalanol A which also inhibits parasite cell growth [31]. PfCRK is crucial to intraerythrocytic development [37]. PfMAP-2 is essential to the asexual cycle of P. falciparum [40]. PfCK $2 \alpha$ has been shown to be crucial to the asexual blood state in Plasmodium [44]. Finally, PfTKL-3 is essential for asexual parasite proliferation in human erythrocytes [45]. Other potential C. parvum kinase targets to consider include those where there is precedence for preferential inhibition of the parasite kinase (including $P$. falciparum and $T$. gondii) over the human orthologue. For example $\operatorname{TgCK} 1 \alpha$ is selectively inhibited by purvalanol B and aminopurvalanol A over host CK enzymes [31]. As well, a range of compound classes that are CDK inhibitors in $P$. falciparum are not effective against the human CDK enzymes tested [34]. Upon analysis of the kinase sequences of $P$. falciparum, T. gondii, and C. parvum herein, we find other non-kinase features that should prompt new drug targets to be selected for study. Several C. parvum kinases include large (and often multiple) inserts and/or $N$ - and $C$-terminal extensions that can be hundreds residues long, but are not conserved and are generally of unknown function. This includes the 40-residue insert in the KD of $C p C D P K 4$ which was structurally identified, herein, as a zinc finger and is suspected to be involved in maintaining its calcium-dependent activity. In addition, there is generally low sequence identity and deviations from the most conserved motifs in the C. parvum protein kinases as compared to its orthologues. All of these factors provide additional parameters that can be explored and potentially exploited for drug design not only for cryptosporidiosis, but also for the other protozoan infections.

The high degree of conservation amongst protein kinases, particularly in the ATP-binding site, challenges 


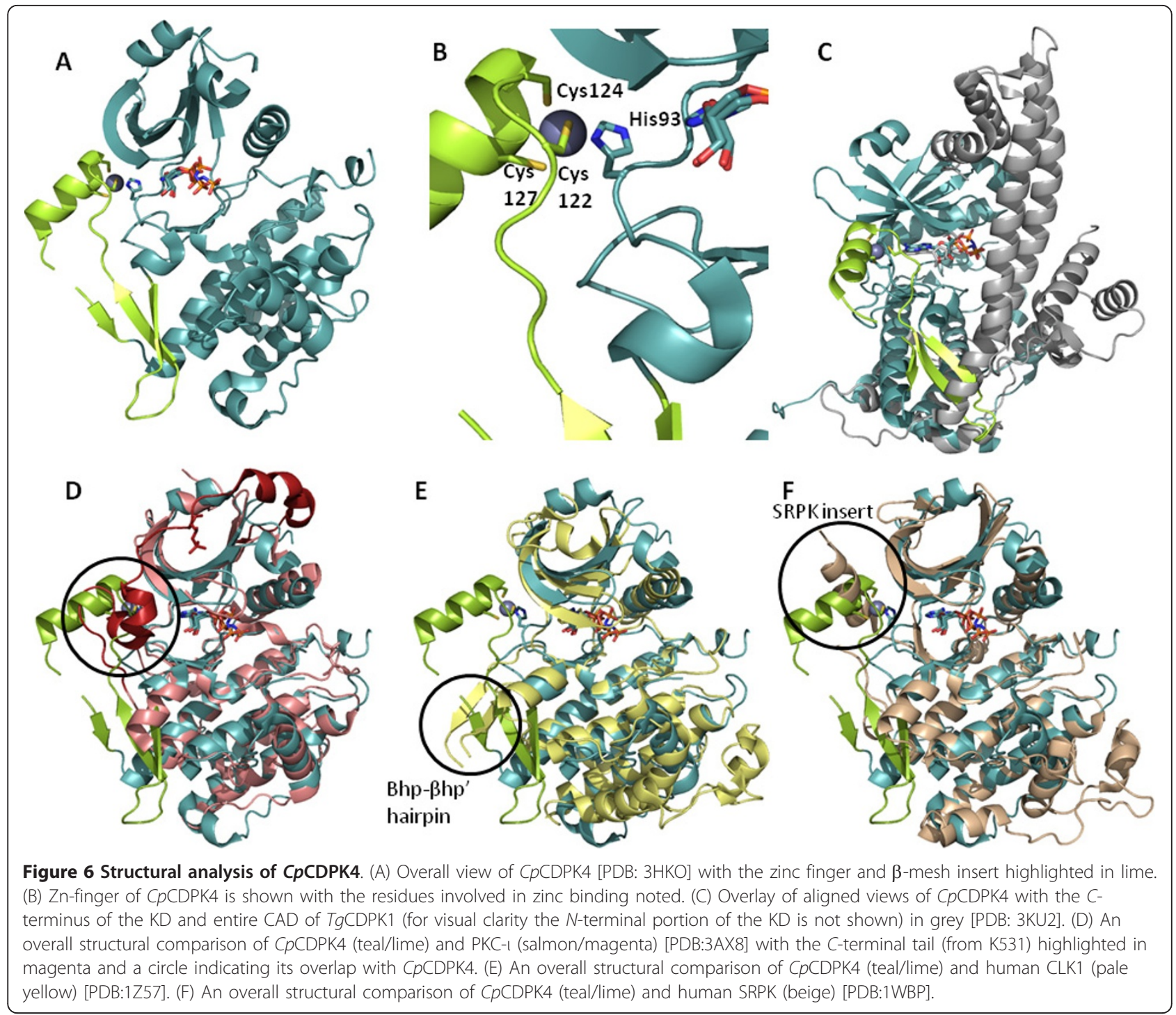

the general goal of finding selective inhibitors. Nevertheless, the adaptation of particular kinases, for example the CDPK enzymes to specific calcium-binding conditions and other regulatory factors, their localization, and their substrate specificity, adds another dimension to the development of inhibitors, thus mitigating the overall kinase drug discovery process. Our structural and biochemical studies of CDPK enzymes further illuminate some of these possibilities with respect to drug design. The exploitation of the gatekeeper in the ATP-binding site shows that opportunities exist for inhibitor design, as is the case for $C p C D P K 1$ which has a glycine-gatekeeper and is inhibited in the nanomolar range by PP1derivatives. Indeed, our crystal structure highlights how one of these inhibitors functions. $C p C D P K 2$ structural analysis shows how a flexible CDK inhibitor can bind and gives a starting point for future development of novel CDPK2 inhibitors. $C p C D P K 4$ has a unique zinc finger and special $\beta$-mesh configuration that may indicate that it can become persistently active, providing an additional mechanism of regulation to the CaMK family that adds another element to the CDPK drug discovery pathway.

For some time it has been known that the CDPK enzymes control crucial functions including transcription, metabolism, ion pumps and channels, and the cytoskeleton [53]. With respect to the protozoan CDPK enzymes, many have been now associated with particular functions. For example, with respect to Plasmodium, PfCDPK1, first identified in the asexual blood stage [54], is involved in the regulation of the motor complex and possibly essential for $P$. falciparum viability $[55,56]$. $P f C D P K 3$ is implicated in sexual stage specific events [57] and more specifically (for $P b C D P K 3$ ) in a signalling 


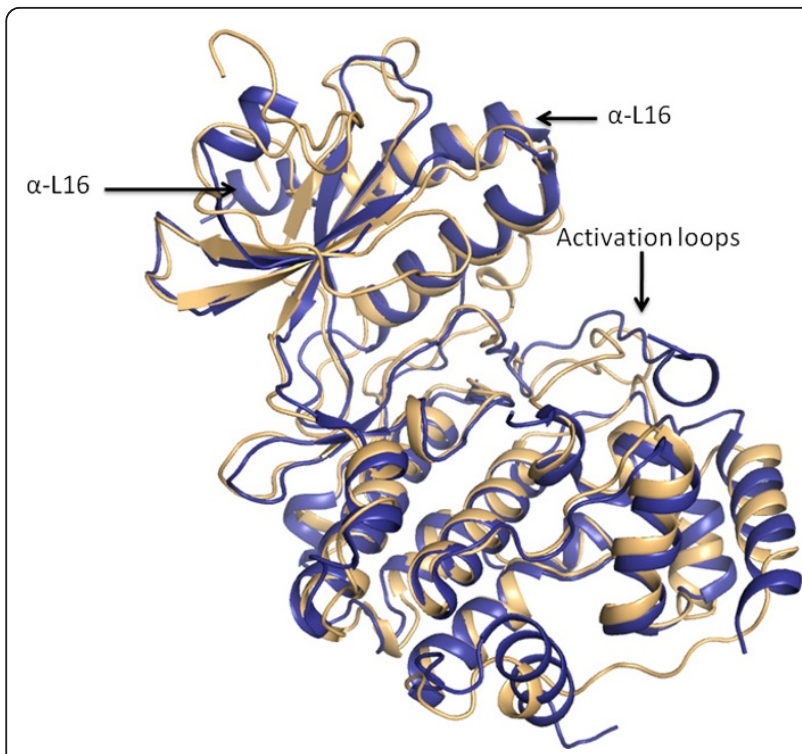

Figure 7 Comparison of CpMAP-1 with the rat ERK2. CpMAP-1 [PDB: 3OZ6] is shown in blue, while ERK2 [PDB: 2ERK] is in beige.

pathway that regulates ookinete penetration of the layer covering the midgut epithelium and a possibly an ookinete-limited essential function [58,59]. PbCDPK4 is essential for male gametogenesis [60]. PfCDPK5 plays an essential role during the blood stage of malaria replication via egress from erythrocytes [61]. PbCDPK6 is critical for the conversion to an invasive $P$. berghei phenotype [62]. In $T$. gondii, knocking out $T g C D P K 1$ by genetic or chemical means indicates that it is an essential regulator of calcium-dependent exocytosis, specifically leading to the inhibition micronemes secretion that results in a block of essential phenotypes including parasite motility, host-cell invasion, and egress. As well, $\mathrm{TgCDPK} 3$ has been suggested to participate in the motility of $T$. gondii through the phosphorylation of glideosome complex member [63]. These results demonstrate the numerous examples of how crucial CDPK enzymes are in both P. falciparum and T. gondii, and as such suggest that $C$. parvum CDPK enzymes may also be associated with essential functions and should be among the targets of cryptosporidiosis drug discovery programs.

Finally, there are huge untapped kinase sources for drug design, as almost a quarter of the C. parvum kinome has no known orthologue outside of Cryptosporidium spp. In addition, the OPK group comprises $40 \%$ of the C. parum kinome, undoubtedly there are unique features within this group to exploit. With numerous kinase inhibitor libraries available, characterization (biochemical and structural) and screening of these kinases may result in the identification of novel targets, potentially without human orthologues, thus greatly facilitating the course of drug discovery. This research can be expedited by considering the kinase classification as presented herein, whereby potential targets are considered not only in the context of their family, but also with respect to their orthologues, a strategy that has streamlined many successful structural genomics projects.

\section{Methods}

\section{Kinome analysis}

To identify protein kinases in the C. parvum genome, a search for various protein kinase domains was conducted using the CryptoDB Version $4.3 \mathrm{http}$ ://www. cryptodb.org domain search utility [21]. Furthermore, a search for the keyword "kinase" was used. This generated a list of 99 candidates. The presence of a protein kinase domain was confirmed by examining their CryptoDB records, leading to elimination of non-protein kinases, regulatory proteins or other non-kinases. The remaining sequences were analysed manually to confirm the presence of a complete catalytic triad resulting in a final list of 73 kinases. Other protein domains and domain architectures were determined by ProSite http:// prosite.expasy.org/[64]. Orthologue group assignments were made by OrthoMCL (Version: 3.0) [65]. The kinase domain sequences of all the $C p \mathrm{PKs}$ and the following structures (PDB: 2WEI, 2QG5, 3HKO, 2QKR, 3EB0, and 3OZ6) were submitted for multiple sequence alignment to the PROMALS3D multiple sequence and structure alignment server http://prodata.swmed.edu/promals3d/ promals3d.php[66]. The alignment results were slightly adjusted manually in the cases of cgd6_4960, cgd2_2310, cgd7_2000, and cgd2_3890, so that the presumed catalytic lysines were aligned (Additional File 2, Table S2). The adjusted alignment was used in the calculation of the phylogenetic inferences by RAxML BlackBox http:// phylobench.vital-it.ch/raxml-bb/index.php[67]. The resulting best scoring ML (maximum-likelihood) tree with branch lengths and support values was submitted to the Interactive Tree of Life Version 2.0.1 website http://itol.embl.de for the rendering of the phylogenetic tree [68]. The same procedure was completed for the analysis of the CDPK family (Additional File 2, Table S3).

\section{Protein expression and purification}

Recombinant samples of $C p$ CDPK1, $C p$ CDPK2, $C p$ CDPK3, and $C p$ CDPK4 (with the following minor truncations (for increased soluble expression): CpCDPK1:M1-E538, CpCDPK2:R186-R667, CpCDPK3: D42-L520, $C p$ CDPK4:L114-R775) were expressed and purified as previously described [27] using entry clones derived from C. parvum strain Iowa genomic DNA (from MR4 - Malaria Research and Reference Reagent Resource Center, Manassas, VA, USA), the Lex bioreactor system (Harbinger Biotechnology and Engineering 
Corp., Toronto, ON) and BL21(DE3)-V2R-pACYC LamP, as the expression host, which includes a plasmid for coexpression of $\lambda$-phosphatase to suppress protein phosphorylation.

\section{Enzymatic characterization and inhibition}

Kinase activity was measured using an NADH-coupled ATPase assay (using pyruvate kinase (PK) and lactate dehydrogenase (LDH)) in a 384-well format based on the method of Dölle and Ziegler [69]. For $\mathrm{IC}_{50}$ determinations, activities were carried out using $10 \mathrm{nM}$ CpCDPK1, $500 \mu \mathrm{M}$ ATP, $500 \mu \mathrm{M}$ Syntide II, and different concentrations of inhibitors $(1 \mathrm{nM}$ to $8 \mu \mathrm{M})$ in 20 $\mathrm{mM}$ Tris, $30 \mathrm{mM} \mathrm{NaCl}, 10 \mathrm{mM} \mathrm{MgCl} 21 \mathrm{mM} \mathrm{CaCl}_{2}, 2$ $\mu \mathrm{g} / \mathrm{ml} \mathrm{BSA}, 10 \mathrm{mM}$ DTT, and $0.01 \%$ Tween 20, pH 7.5. ADP production was measured in BioTek Senergy2 plate reader, using LDH/PK coupled assay with $150 \mu \mathrm{M}$ NADH, $300 \mu \mathrm{M}$ PEP and LDH/PK mix from Sigma (with 3 units of $\mathrm{LDH} / \mathrm{mL}$ ). The reaction was initiated with the addition of $C p C D P K 1$. Initial rates were calculated (for 3 to 10 minutes) and data were analyzed using SigmaPlot 9.

\section{Protein crystallization, data collection, and refinement}

Apo $C p$ CDPK1 was crystallized from hanging drops in $0.1 \mathrm{M}$ Tris pH 7.6, 20\% PEG 8000, and 0.38 M ammonium sulfate. The crystal was flash-cooled in liquid nitrogen using mother liquor supplemented with $25 \%$ ethylene glycol as a cryoprotectant. Data were collected at APS 23ID-B http://www.gmca.anl.gov and processed with XDS indexing and scaling software [70]. $C p$ CDPK1 with the 3MB-PP1 derivative bound was crystallized in sitting drop format from 25\% PEG 3350 and $0.1 \mathrm{M}$ BisTris $\mathrm{pH}$ 5.5. The crystal was flash-cooled in liquid nitrogen. Data were collected at SLS X10SA http://x10sa.web.psi.ch. Apo $C p$ CDPK2 was crystallized from hanging drops in $2.5 \mathrm{M}$ sodium formate and 0.2 $\mathrm{M}$ BisTris propane $\mathrm{pH}$ 7.0. Data from the flash-cooled crystal were collected at APS 17ID http://www.sbc.anl. gov and processed with the HKL3000 indexing and scaling software [71]. Cp CDPK2 with indirubin E804 bound was crystallized from a solution of $18 \%$ PEG 3350, $0.1 \mathrm{M}$ ammonium sulfate, $0.1 \mathrm{M}$ sodium cacodylate and $5 \mathrm{mM}$ indirubin E804. Data for the crystal flash-cooled in liquid nitrogen were collected using a home source Rigaku FRE Superbright rotating anode with an RAXIS IV plate reader. Data were processed using the HKL2000 indexing and scaling software [72]. CpCDPK4 (with $5 \mathrm{mM}$ AMPPNP (phosphoaminophosphonic acid-adenylate ester) and $2 \mathrm{mM} \mathrm{MgCl}_{2}$ ) was crystallized in $0.1 \mathrm{M}$ Hepes pH 7.5, 25\% PEG 3350, and $0.2 \mathrm{M} \mathrm{NaCl}$. Data from flash-cooled crystals (with $25 \%$ glycerol as a cryoprotectant) were collected on the A1 beamline at CHESS http://www.chess.cornell.edu and processed with HKL2000 [72]. CpMAP-1 crystals formed in 20\% PEG 3350, $0.1 \mathrm{M}$ magnesium acetate, 4 mM AMPPNP and $4 \mathrm{mM}$ TCEP-HCl (tris(2-carboxyethyl)phosphine hydrochloride salt) by the hanging drop method. Crystals were flash-cooled in liquid nitrogen. Data were collected at APS 19ID http://www. sbc.anl.gov and processed with the HKL3000 indexing and scaling software [71]. Each structure was solved by molecular replacement using modified homology models created with the FFAS03 program [73]. The following models were used: $C p$ CDPK1 used PDB: 2QG5, $C p$ CDPK2 used 1A06, $C p$ CDPK4 used PDB: 3DFA, and $C p$ MAP-1 used PDB: 2B9F. The structures were refined by iterative rounds of manual building in Coot 3.0 and refinement using the ccp4i program refmac5 [74]. All structures were refined with good statistics and geometry, checked with MOLPROBITY [75] and with no outliers in the Ramachandran plot. Final statistics and data information for each structure can be found in Table 4. Figures for structural models were created using the Pymol visualization software http:// www.pymol.org and Microsoft PowerPoint.

\section{Additional material}

Additional file 1: Table S1 - Classification of the $C$. parvum protein

kinases. Table listing the 73 protein kinases from C. parvum, showing the families, PDB depositions, and orthologues from data compiled from the sequence similarity within the kinase domain, their predicted

orthologues, and a maximum likelihood tree from a rapid bootstrap analysis.

Additional file 2: Table S2 - Alignnments of $C$. parvum kinases. Exce sheet showing the alignment of the 73 protein kinase catalytic domains, as calculated by PROMALS3D multiple sequence and structure alignment server http://prodata.swmed.edu/promals3d/promals3d.php. Table S3 Alignment of select CaMK enzymes. Excel sheet showing the alignment of the kinase domains of select CaMK family members, as calculated by PROMALS3D multiple sequence and structure alignment server http:// prodata.swmed.edu/promals3d/promals3d.php.

Additional file 3: Table S4 - $\mathrm{N}$-terminal latch of select CDPK enzymes. Table showing a partial sequence alignment of CDPK enzymes highlighting conserved $\mathrm{N}$-terminal motifs implicated in binding the $\mathrm{N}$ terminal latch. Table S5 - Gatekeepers in the C. parvum CDPK enzymes.

\section{Acknowledgements and Funding}

This work was supported by the Canadian Institutes for Health Research, the Canadian Foundation for Innovation, Genome Canada through the Ontario Genomics Institute, GlaxoSmithKline, the Knut and Alice Wallenberg Foundation, the Ontario Innovation Trust, the Ontario Ministry for Research and Innovation, Merck \& Co., Inc., the Novartis Research Foundation, the Petrus and Augusta Hedlund's Foundation, the Swedish Agency for Innovation Systems, the Swedish Foundation for Strategic Research, and the Wellcome Trust (all to the Structural Genomics Consortium, registered charity number 1097737).

\section{Author details}

${ }^{1}$ Structural Genomics Consortium, University of Toronto, MaRS South Tower, Floor 7, 101 College St, Toronto, Ontario M5G 1L7, Canada. ${ }^{2}$ Structural Genomics Consortium, University of Oxford, Old Road Research Building, Roosevelt Drive, Oxford OX3 7DQ, UK. ${ }^{3}$ Howard Hughes Medical Institute, 
Department of Cellular and Molecular Pharmacology, University of California at San Francisco, San Francisco, California 94158, USA.

\section{Authors' contributions}

JDA carried out the kinome analysis, drafted the manuscript, and prepared the figures. AKW analyzed the structural data and wrote part of the structural analysis. AKW, WQ, and WL collected diffraction data and solved all of the structures (except for [PDB: 2WEI]). AAH, GS, GAW, PF, IC, and MV measured the activity of the CDPK enzymes. $Y Z$ and MS also participated in the kinase domain analysis. MA, YL, and $\mathrm{AH}$ expressed, purified, and crystallized the CDPK and MAP-1 enzymes. OF and OK screened CpCDPK1 against the inhibitor library. AR and FvD crystallized and solved the CPCDPK1 structure with the PP1 derivative [PDB: $2 \mathrm{WEI}$. FM, $J$, and IK cloned the CDPK and MAP-1 constructs. CZ and KS selected and synthesized PP1 inhibitors. TH analyzed the sequence alignment of CDPK enzymes and identified key motifs. $\mathrm{RH}$ conceived the study, initiated the kinome analysis, and helped draft the manuscript. All authors read and approved the final manuscript.

Received: 5 May 2011 Accepted: 30 September 2011

Published: 30 September 2011

\section{References}

1. Putignani $L$, Menichella D: Global distribution, public health and clinical impact of the protozoan pathogen cryptosporidium. Interdiscip Perspect Infect Dis 2010, 2010.

2. Chalmers RM, Davies AP: Minireview: clinical cryptosporidiosis. Exp Parasitol 2010, 124(1):138-146.

3. Checkley W, Epstein LD, Gilman RH, Black RE, Cabrera L, Sterling CR: Effects of Cryptosporidium parvum infection in Peruvian children: growth faltering and subsequent catch-up growth. Am J Epidemiol 1998, 148(5):497-506.

4. Chen XM, Keithly JS, Paya CV, LaRusso NF: Cryptosporidiosis. N Engl J Med 2002, 346(22):1723-1731.

5. Sallon S, Deckelbaum RJ, Schmid II, Harlap S, Baras M, Spira DT: Cryptosporidium, malnutrition, and chronic diarrhea in children. Am J Dis Child 1988, 142(3):312-315.

6. Savioli L, Smith H, Thompson A: Giardia and Cryptosporidium join the 'Neglected Diseases Initiative'. Trends Parasitol 2006, 22(5):203-208.

7. Cabada MM, White AC Jr: Treatment of cryptosporidiosis: do we know what we think we know? Curr Opin Infect Dis 2010, 23(5):494-499.

8. Fedorov O, Muller S, Knapp S: The (un)targeted cancer kinome. Nat Chem Biol 2010, 6(3):166-169.

9. Edwards AM, Isserlin R, Bader GD, Frye SV, Willson TM, Yu FH: Too many roads not taken. Nature 2011, 470(7333):163-165.

10. Umejiego NN, Li C, Riera T, Hedstrom L, Striepen B: Cryptosporidium parvum IMP dehydrogenase: identification of functional, structural, and dynamic properties that can be exploited for drug design. The Journal of biological chemistry 2004, 279(39):40320-40327.

11. Ctrnacta V, Fritzler JM, Surinova M, Hrdy I, Zhu G, Stejskal F: Efficacy of Sadenosylhomocysteine hydrolase inhibitors, D-eritadenine and (S)-DHPA, against the growth of Cryptosporidium parvum in vitro. Exp Parasitol 2010, 126(2):113-116

12. Artz JD, Dunford JE, Arrowood MJ, Dong A, Chruszcz M, Kavanagh KL, Minor W, Russell RG, Ebetino FH, Oppermann U, et al: Targeting a uniquely nonspecific prenyl synthase with bisphosphonates to combat cryptosporidiosis. Chemistry \& biology 2008, 15(12):1296-1306.

13. Murphy RC, Ojo KK, Larson ET, Castellanos-Gonzalez A, Perera BG, Keyloun KR, Kim JE, Bhandari JG, Muller NR, Verlinde CL, et al: Discovery of Potent and Selective Inhibitors of Calcium-Dependent Protein Kinase 1 (CDPK1) from C. parvum and T. gondii. ACS Med Chem Lett 2010, 1(7):331-335.

14. Manning G, Plowman GD, Hunter T, Sudarsanam S: Evolution of protein kinase signaling from yeast to man. Trends Biochem Sci 2002, 27(10):514-520.

15. Doerig C, Abdi A, Bland N, Eschenlauer S, Dorin-Semblat D, Fennell C, Halbert J, Holland Z, Nivez MP, Semblat JP, et al: Malaria: targeting parasite and host cell kinomes. Biochim Biophys Acta 2010, 1804(3):604-612

16. Anamika, Srinivasan N, Krupa A: A genomic perspective of protein kinases in Plasmodium falciparum. Proteins 2005, 58(1):180-189.
17. Ward $P$, Equinet $L$, Packer J, Doerig C: Protein kinases of the human malaria parasite Plasmodium falciparum: the kinome of a divergent eukaryote. BMC Genomics 2004, 5(1):79.

18. Anamika K, Srinivasan N: Comparative kinomics of Plasmodium organisms: unity in diversity. Protein Pept Lett 2007, 14(6):509-517.

19. Peixoto L, Chen F, Harb OS, Davis PH, Beiting DP, Brownback CS, Ouloguem D, Roos DS: Integrative genomic approaches highlight a family of parasite-specific kinases that regulate host responses. Cell Host Microbe 2010, 8(2):208-218.

20. Hanks SK, Hunter T: Protein kinases 6. The eukaryotic protein kinase superfamily: kinase (catalytic) domain structure and classification. Faseb J 1995, 9(8):576-596.

21. Heiges M, Wang H, Robinson E, Aurrecoechea C, Gao X, Kaluskar N, Rhodes P, Wang S, He CZ, Su Y, et al: CryptoDB: a Cryptosporidium bioinformatics resource update. Nucleic acids research 2006, , 34 Database: D419-422.

22. Taylor HM, McRobert L, Grainger M, Sicard A, Dluzewski AR, Hopp CS, Holder AA, Baker DA: The malaria parasite cyclic GMP-dependent protein kinase plays a central role in blood-stage schizogony. Eukaryot Cell 2010, 9(1):37-45.

23. McRobert L, Taylor CJ, Deng W, Fivelman QL, Cummings RM, Polley SD, Billker O, Baker DA: Gametogenesis in malaria parasites is mediated by the cGMP-dependent protein kinase. PLoS biology 2008, 6(6):e139.

24. Billker O, Lourido S, Sibley LD: Calcium-dependent signaling and kinases in apicomplexan parasites. Cell Host Microbe 2009, 5(6):612-622.

25. Nagamune K, Sibley LD: Comparative genomic and phylogenetic analyses of calcium ATPases and calcium-regulated proteins in the apicomplexa. Mol Biol Evol 2006, 23(8):1613-1627.

26. Kato K, Sudo A, Kobayashi K, Tohya Y, Akashi H: Characterization of Plasmodium falciparum protein kinase 2. Molecular and biochemical parasitology 2008, 162(1):87-95.

27. Wernimont AK, Artz JD, Finerty P Jr, Lin YH, Amani M, Allali-Hassani A Senisterra G, Vedadi M, Tempel W, Mackenzie F, et al: Structures of apicomplexan calcium-dependent protein kinases reveal mechanism of activation by calcium. Nat Struct Mol Biol 2010, 17(5):596-601.

28. Wernimont AK, Amani M, Qiu W, Pizarro JC, Artz JD, Lin YH, Lew J, Hutchinson A, Hui R: Structures of parasitic CDPK domains point to a common mechanism of activation. Proteins 2010 [http://onlinelibrary.wiley. com/doi/10.1002/prot.22919/abstract; jsessionid=2EDC0241E9953E7BA75A3C8FB33BF4FA.d02t03].

29. Chen F, Mackey AJ, Stoeckert CJ Jr, Roos DS: OrthoMCL-DB: querying a comprehensive multi-species collection of ortholog groups. Nucleic Acids Res 2006, 34 Database: D363-368.

30. Knockaert M, Gray N, Damiens E, Chang YT, Grellier P, Grant K, Fergusson D, Mottram J, Soete M, Dubremetz JF, et al: Intracellular targets of cyclindependent kinase inhibitors: identification by affinity chromatography using immobilised inhibitors. Chem Biol 2000, 7(6):411-422.

31. Donald RG, Zhong T, Meijer L, Liberator PA: Characterization of two $T$. gondii CK1 isoforms. Mol Biochem Parasitol 2005, 141(1):15-27.

32. Miranda-Saavedra D, Barton GJ: Classification and functional annotation of eukaryotic protein kinases. Proteins 2007, 68(4):893-914.

33. Dorin D, Semblat JP, Poullet P, Alano P, Goldring JP, Whittle C, Patterson S, Chakrabarti D, Doerig C: PfPK7, an atypical MEK-related protein kinase, reflects the absence of classical three-component MAPK pathways in the human malaria parasite Plasmodium falciparum. Molecular microbiology 2005, 55(1):184-196.

34. Geyer JA, Prigge ST, Waters NC: Targeting malaria with specific CDK inhibitors. Biochim Biophys Acta 2005, 1754(1-2):160-170.

35. Caridha D, Kathcart AK, Jirage D, Waters NC: Activity of substituted thiophene sulfonamides against malarial and mammalian cyclin dependent protein kinases. Bioorg Med Chem Lett 2010, 20(13):3863-3867.

36. Jirage $D$, Chen Y, Caridha D, O'Neil MT, Eyase F, Witola WH, Mamoun CB, Waters NC: The malarial CDK Pfmrk and its effector PfMAT1 phosphorylate DNA replication proteins and co-localize in the nucleus. Molecular and biochemical parasitology 2010, 172(1):9-18.

37. Halbert J, Ayong L, Equinet L, Le Roch K, Hardy M, Goldring D, Reininger L, Waters N, Chakrabarti D, Doerig C: A Plasmodium falciparum transcriptional cyclin-dependent kinase-related kinase with a crucial role in parasite proliferation associates with histone deacetylase activity. Eukaryot Cell 2010, 9(6):952-959. 
38. Holton S, Merckx A, Burgess D, Doerig C, Noble M, Endicott J: Structures of $P$. falciparum PfPK5 test the CDK regulation paradigm and suggest mechanisms of small molecule inhibition. Structure 2003, 11(11):1329-1337.

39. Dorin D, Alano P, Boccaccio I, Ciceron L, Doerig C, Sulpice R, Parzy D: An atypical mitogen-activated protein kinase (MAPK) homologue expressed in gametocytes of the human malaria parasite Plasmodium falciparum. Identification of a MAPK signature. The Journal of biological chemistry 1999, 274(42):29912-29920.

40. Dorin-Semblat D, Quashie N, Halbert J, Sicard A, Doerig C, Peat E, RanfordCartwright L: Functional characterization of both MAP kinases of the human malaria parasite Plasmodium falciparum by reverse genetics. Molecular microbiology 2007, 65(5):1170-1180.

41. Droucheau E, Primot A, Thomas V, Mattei D, Knockaert M, Richardson C, Sallicandro P, Alano P, Jafarshad A, Baratte B, et al: Plasmodium falciparum glycogen synthase kinase-3: molecular model, expression, intracellular localisation and selective inhibitors. Biochim Biophys Acta 2004, 1697(12):181-196.

42. Li JL, Targett GA, Baker DA: Primary structure and sexual stage-specific expression of a LAMMER protein kinase of Plasmodium falciparum. International journal for parasitology 2001, 31(4):387-392.

43. Kannan N, Neuwald AF: Evolutionary constraints associated with functional specificity of the CMGC protein kinases MAPK, CDK, GSK, SRPK, DYRK, and CK2alpha. Protein science: a publication of the Protein Society 2004, 13(8):2059-2077.

44. Holland Z, Prudent R, Reiser JB, Cochet C, Doerig C: Functional analysis of protein kinase CK2 of the human malaria parasite Plasmodium falciparum. Eukaryot Cell 2009, 8(3):388-397.

45. Abdi A, Eschenlauer S, Reininger L, Doerig C: SAM domain-dependent activity of PfTKL3, an essential tyrosine kinase-like kinase of the human malaria parasite Plasmodium falciparum. Cell Mol Life Sci 2010, 67(19):3355-3369.

46. Schneider AG, Mercereau-Puijalon O: A new Apicomplexa-specific protein kinase family: multiple members in Plasmodium falciparum, all with an export signature. BMC Genomics 2005, 6(1):30.

47. Dorin D, Le Roch K, Sallicandro P, Alano P, Parzy D, Poullet P, Meijer L, Doerig C: Pfnek-1, a NIMA-related kinase from the human malaria parasite Plasmodium falciparum Biochemical properties and possible involvement in MAPK regulation. Eur J Biochem 2001, 268(9):2600-2608.

48. Farber PM, Graeser R, Franklin RM, Kappes B: Molecular cloning and characterization of a second calcium-dependent protein kinase of Plasmodium falciparum. Molecular and biochemical parasitology 1997, 87(2):211-216.

49. Takimura T, Kamata K, Fukasawa K, Ohsawa H, Komatani H, Yoshizumi T, Takahashi I, Kotani H, Iwasawa Y: Structures of the PKC-iota kinase domain in its ATP-bound and apo forms reveal defined structures of residues 533-551 in the C-terminal tail and their roles in ATP binding. Acta Crystallogr D Biol Crystallogr 2010, 66(Pt 5):577-583.

50. Bullock AN, Das S, Debreczeni JE, Rellos P, Fedorov O, Niesen FH, Guo K, Papagrigoriou E, Amos AL, Cho S, et al: Kinase domain insertions define distinct roles of CLK kinases in SR protein phosphorylation. Structure 2009, 17(3):352-362.

51. Ngo JC, Chakrabarti S, Ding JH, Velazquez-Dones A, Nolen B, Aubol BE, Adams JA, Fu XD, Ghosh G: Interplay between SRPK and Clk/Sty kinases in phosphorylation of the splicing factor ASF/SF2 is regulated by a docking motif in ASF/SF2. Mol Cell 2005, 20(1):77-89.

52. Donald RG, Allocco J, Singh SB, Nare B, Salowe SP, Wiltsie J, Liberator PA: Toxoplasma gondii cyclic GMP-dependent kinase: chemotherapeutic targeting of an essential parasite protein kinase. Eukaryot Cell 2002, 1(3):317-328.

53. Harmon AC, Gribskov M, Harper JF: CDPKs - a kinase for every Ca2+ signal? Trends Plant Sci 2000, 5(4):154-159.

54. Zhao Y, Kappes B, Franklin RM: Gene structure and expression of an unusual protein kinase from Plasmodium falciparum homologous at its carboxyl terminus with the EF hand calcium-binding proteins. The Journal of biological chemistry 1993, 268(6):4347-4354.

55. Green JL, Rees-Channer RR, Howell SA, Martin SR, Knuepfer E, Taylor HM, Grainger M, Holder AA: The motor complex of Plasmodium falciparum: phosphorylation by a calcium-dependent protein kinase. The Journal of biological chemistry 2008, 283(45):30980-30989.
56. Kato N, Sakata T, Breton G, Le Roch KG, Nagle A, Andersen C, Bursulaya B, Henson K, Johnson J, Kumar KA, et al: Gene expression signatures and small-molecule compounds link a protein kinase to Plasmodium falciparum motility. Nat Chem Biol 2008, 4(6):347-356.

57. Li JL, Baker DA, Cox LS: Sexual stage-specific expression of a third calcium-dependent protein kinase from Plasmodium falciparum. Biochim Biophys Acta 2000, 1491(1-3):341-349.

58. Ishino T, Orito Y, Chinzei Y, Yuda M: A calcium-dependent protein kinase regulates Plasmodium ookinete access to the midgut epithelial cell. Molecular microbiology 2006, 59(4):1175-1184.

59. Siden-Kiamos I, Ecker A, Nyback S, Louis C, Sinden RE, Billker O: Plasmodium berghei calcium-dependent protein kinase 3 is required for ookinete gliding motility and mosquito midgut invasion. Molecular microbiology 2006, 60(6):1355-1363.

60. Billker O, Dechamps S, Tewari R, Wenig G, Franke-Fayard B, Brinkmann V: Calcium and a calcium-dependent protein kinase regulate gamete formation and mosquito transmission in a malaria parasite. Cell 2004, 117(4):503-514.

61. Dvorin JD, Martyn DC, Patel SD, Grimley JS, Collins CR, Hopp CS, Bright AT, Westenberger S, Winzeler E, Blackman MJ, et al: A plant-like kinase in Plasmodium falciparum regulates parasite egress from erythrocytes. Science 2010, 328(5980):910-912.

62. Coppi A, Tewari R, Bishop JR, Bennett BL, Lawrence R, Esko JD, Billker O, Sinnis P: Heparan sulfate proteoglycans provide a signal to Plasmodium sporozoites to stop migrating and productively invade host cells. Cell host \& microbe 2007, 2(5):316-327.

63. Sugi T, Kato K, Kobayashi K, Pandey K, Takemae H, Kurokawa H, Tohya Y, Akashi H: Molecular analyses of Toxoplasma gondii calmodulin-like domain protein kinase isoform 3. Parasitol Int 2009, 58(4):416-423.

64. Sigrist CJ, Cerutti L, de Castro E, Langendijk-Genevaux PS, Bulliard V, Bairoch A, Hulo N: PROSITE, a protein domain database for functional characterization and annotation. Nucleic acids research 2010, , 38 Database: D161-166.

65. Li L, Stoeckert CJ Jr, Roos DS: OrthoMCL: identification of ortholog groups for eukaryotic genomes. Genome research 2003, 13(9):2178-2189.

66. Pei J, Kim BH, Grishin NV: PROMALS3D: a tool for multiple protein sequence and structure alignments. Nucleic acids research 2008, 36(7):2295-2300

67. Stamatakis A, Hoover P, Rougemont J: A rapid bootstrap algorithm for the RAxML Web servers. Syst Biol 2008, 57(5):758-771.

68. Letunic I, Bork P: Interactive Tree Of Life (iTOL): an online tool for phylogenetic tree display and annotation. Bioinformatics 2007, 23(1):127-128.

69. Dolle C, Ziegler M: Application of a coupled enzyme assay to characterize nicotinamide riboside kinases. Anal Biochem 2009, 385(2):377-379.

70. Kabsch W: Automatic Processing of Rotation Diffraction Data from Crystals of Initially Unknown Symmetry and Cell Constants. J App/ Crystallogr 1993, 26:795-800.

71. Minor W, Cymborowski M, Otwinowski Z, ChruszCz M: HKL-3000: the integration of data reduction and structure solution - from diffraction images to an initial model in minutes. Acta Crystallogr D 2006, 62:859-866.

72. Otwinowski Z, Minor W: Processing of X-ray diffraction data collected in oscillation mode. Method Enzymol 1997, 276:307-326.

73. Jaroszewski L, Rychlewski L, Li ZW, Li WZ, Godzik A: FFAS03: a server for profile-profile sequence alignments. Nucleic Acids Research 2005, 33: W284-W288.

74. Bailey S: The Ccp4 Suite - Programs for Protein Crystallography. Acta Crystallogr D 1994, 50:760-763.

75. Davis IW, Murray LW, Richardson JS, Richardson DC: MolProbity: structure validation and all-atom contact analysis for nucleic acids and their complexes. Nucleic Acids Research 2004, 32:W615-W619.

doi:10.1186/1471-2164-12-478

Cite this article as: Artz et al:: The Cryptosporidium parvum Kinome. BMC Genomics 2011 12:478. 